\title{
Plantain based dough meal: nutritional property, antioxidant activity and dyslipidemia ameliorating potential in high- fat induced rats
}

Timilehin David Oluwajuyitan * (D), Oluwole Steve ljarotimi and Tayo Nathaniel Fagbemi

\begin{abstract}
s
Background: Dyslipidemia is an aberrant rise in blood lipids due to diet and lifestyle. It has implicated as the major risk factor for developing hypertension among other diseases. This study was designed to evaluate plantain based dough meal nutritional property, antioxidant activity and dyslipidemia ameliorating potential in high-fat induced rats.

Methods: The flour blends, i.e., PSC (Plantain 70\%, Soycake 30\%), PSR (Plantain 65\%, Soycake 30\%, Rice-bran 5\%), PSO (Plantain 65\%, Soycake 30\%, Oat-bran 5\%), PSRO (Plantain 60\%, Soycake 30\%, Rice-bran 5\%, Oat-bran 5\%) and controls (100\% Plantain flour \& Cerolina) were evaluated for chemical, antioxidants and antihyperlipidemia.

Results: Protein, fiber and energy composition varied from $2.2-4.97 \mathrm{~g} / 100 \mathrm{~g}, 16.44-19.59 \mathrm{~g} / 100 \mathrm{~g}$ and 369.7-385.5 $\mathrm{kcal} / 100 \mathrm{~g}$, respectively. Essential amino acid index and predicted-biological values of the foods ranged from 68.31$76.31 \%$ and $62.19-71.48 \%$, respectively. Phenolic profiles (mg/g) were gallic acid (25.33-31.26), caffeic acid (2.754.61), ferulic acid (5.16-12.73), luteolin (16.31-23.60), kaempferol (21.51-30.64), quercetin (24.28-37.13), chlorogenic acid (42.25-59.78), myricetin (28.41-38.41), 3,5-dicaffeoylquinic acid (27.17-41.59) and 4, 5-dicaffeoylquinic acid (39.96-51.28). The antioxidant activity of PSRO on ABTS, DPPH, FRAP, Fe ${ }^{2+}$ chelation and OH free radicals was higher than other foods. Atherogenic index, coronary risk index and log (TG/HDL-conc.) of rats fed on experimental foods were lower than recommended values.
\end{abstract}

Conclusion: The study established that PSRO had higher antioxidant and anti-hyperlipidemia properties; hence, it may be suitable as a functional food.

Keywords: Functional food, Nutritional composition, Phenolic compounds, Antioxidant activity, Antihyperlipidemic

\section{Introduction}

Hypertension is a major risk factor for morbidity and premature mortality in developed and developing countries; hence, it is considered a global public health problem [1]. High concentration of Low-Density Lipoprotein cholesterol (LDL-C) in the blood has been implicated as the major risk factor for developing hypertension [2].

\footnotetext{
* Correspondence: tdoluwajuyitan@futa.edu.ng

Department of Food Science and Technology, Federal University of Technology, Akure, Nigeria
}

\section{Springer Open}

(c) The Author(s). 2021 Open Access This article is licensed under a Creative Commons Attribution 4.0 International License which permits use, sharing, adaptation, distribution and reproduction in any medium or format, as long as you give appropriate credit to the original author(s) and the source, provide a link to the Creative Commons licence, and indicate if changes were made. The images or other third party material in this article are included in the article's Creative Commons licence, unless indicated otherwise in a credit line to the material. If material is not included in the article's Creative Commons licence and your intended use is not permitted by statutory regulation or exceeds the permitted use, you will need to obtain permission directly from the copyright holder. To view a copy of this licence, visit http://creativecommons.org/licenses/by/4.0/.

Hypertension is defined as a systolic blood pressure (SBP) $\geq 140 \mathrm{mmHg}$ and/or a diastolic blood pressure (DBP) $\geq 90 \mathrm{mmHg}$ [3], and it is commonly referred to as a silent killer. The prevalence of hypertension in traditional African communities, where it is once rare, is increasing [4], due to both sedentary lifestyle and regular intakes of processed diets high in salt and animal fats [5-7]. This unhealthy dietary intake has increased the prevalence of obesity, atherosclerosis-related diseases [8] and impaired fasting glucose [9], which are the major risk factors for hypertension [10]. Evidence has shown 
that synthetic anticholesterol and antihypertensive agents are frequently used to lower high blood cholesterol and pressure; however, these had recently been implicated with many side effects $[11,12]$. In recent time, efforts have been shifted towards production of therapeutic diets high in antioxidants and fiber with non-side effects to prevent high blood cholesterol and associated diseases [12-14]. Functional foods are those modified food ingredients or conventional food, which are consumed as part of a normal diet but provides physiological benefits and/or reducing the risk of chronic disease beyond basic nutritional functions [15].

The health promoting properties of functional foods are attributed to bioactive components such as phytochemicals, peptides, fibers, etc. [16]. In recent time, there is increased in the interest of functional foods consumption patterns with the aim of reducing risk of hypertension and other chronic diseases [13]. Diets from plant origin are rich in antioxidant phytochemicals with the abilities to scavenge free radicals, and thereby inhibiting the deleterious effects of oxidative stress [17]. The drives to search for novel, cheap and effective plant-based foods with antioxidant and blood cholesterol and pressure lowering potentials become necessary. It is evidence that regular consumption of phenolic compounds present in plant-based foods is associated with health benefits in man [13]. Some phenolic compounds in foods have antioxidants activities, thereby contributing to a reduction in the risk of arteriosclerosis and cardiovascular diseases $[18,19]$. Studies have shown that phenolic compounds such as gallic acid and quercetin have the potential to prevent hypertension, diabetes and other degenerative chronic diseases $[18,19]$.

Plantain (Musa ABB) is widely cultivated in Nigeria and consumed as a cheap source of energy. Nutritionally, plantain particularly unripe is a good source of carbohydrate, dietary fiber, irons, potassium and vitamins [20, 21], and regular consumption of plantain helps to prevent diabetes, hypertension and anaemia due to its high resistant starch content and low glycemic index property [22-24]. Plantain flour has been successfully added to cereals to produce bread [25], spaghetti [26] and other food products, demonstrating that its addition results in a higher resistant starch content and a lower starch digestion rate. In Southwest Nigeria, it is consumed in form of a dough meal - a prepared cooked paste from traditional diets/ plants that is food sustainable, safe, nutritious, culturally acceptable and available all year round.

Soycake (Glycine $\max \mathrm{L}$ ) is a by-product generated during oil extraction of soybeans oil. Soybean cake is high in protein and can be used in food formulation to prevent protein-energy malnutrition. Soybean cake is used as a protein supplement and has a good number of advantages ranging from low cost, readily available and health benefits in man. The application of soycake in food formulation particularly with unripe plantain flour has recently been reported [27-29].

Rice bran (Oryza sativa L) is a by-product of rice milling; and it is rich in tocotrienol and ability to reduce total cholesterol and low-density lipoprotein (bad cholesterol). Hence, tocotrienol is used to prevent the risk of heart disease in man [30,31]. Evidences have shown that blending rice-bran into animal formulations reduced cholesterol levels [32, 33].

Oat bran (Avena sativa L.) is a by-product of oatmeal and it is reported to contain $\beta$-glucan which significantly increasing blood concentrations of high-density lipoprotein cholesterol [34]. Evidence has established that regular consumption of oat-meals is beneficial to manage hypertension due to its fiber content and bioactive phytochemicals [35]. The $\beta$-glucan in oats plays an important role in preventing and managing diabetes by slowing down carbohydrate digestion and absorption of glucose in the small intestine [36].

Recently, efforts have been shifted towards production of functional foods from local food materials to manage chronic degenerative diseases $[29,37,38]$. Despite all these efforts, there is scanty information on the combination of plantain, oat bran, rice bran and soycake flour in food formulation. Hence, this study was aimed to produce and evaluates nutritional composition, bioactive phytochemicals, antioxidants and anti-cholesterol potential of diets formulated from plantain, oat-bran, ricebran and soycake.

\section{Materials and methods}

\section{Sources of food sample}

The unripe plantains were obtained from Teaching and Research (T\&R) Farm, Federal University of Technology, Akure (FUTA), Nigeria; defatted soybean cake were obtained from Rom Mill Factory, Ibadan, Nigeria; rice-bran were obtained from Igbimo Rice Processing Company, Aisegba-Ekiti, Nigeria and oat-bran were obtained from Richardson Milling Limited, Portage La Prairie, Manitoba (MB), respectively.

The food materials were obtained during the first quarter of the year, authenticated at the Department of Crop, Soil and Pest Management, FUTA, Nigeria (FUTA/CSP/AUT/2019/0087) and stored at room temperature in an air tight container after processing into flour prior to analysis.

\section{Processing of flour and food formulations}

The plantain flour was processed using method described by Mepba et al. [39] with slight modification. The plantains were manually peeled and sliced into $1 \mathrm{~cm}$ thick pieces, blanched $\left(100^{\circ} \mathrm{C}, 10 \mathrm{~min}\right.$. $)$ in sodium 
hydrogen sulphate $\left(1.25 \% \mathrm{NaHSO}_{3}\right)$ solution to prevent browning and drained. The food samples, i.e., soycake, rice-bran, oat-bran and blanched plantain were dried in hot-air oven (Plus11 Sanyo Gallenkamp PLC, Loughborough, Leicestershire, UK) at $60^{\circ} \mathrm{C}$ for $24 \mathrm{~h}$, milled into flour (Laboratory blender (Model KM 901D; Kenwood Electronic, Hertfordshire, UK) and sieved through 200 $\mathrm{mm}$ mesh sieve (British Standard) to obtain experimental flour samples.

The flour samples, i.e., plantain, soycake, rice-bran and oat-bran flour, were blended with reference to $14 \mathrm{~g} /$ day protein and $5 \mathrm{~g} /$ day fibre (i.e., $25 \%$ of recommended daily intakes of an adult requirements) using Response Surface Methodology. The flour samples were blended as follows: PSC (Plantain 70\%, Soycake 30\%), PSR (Plantain $65 \%$, Soycake $30 \%$, Rice bran $5 \%$ ), PSO (Plantain $65 \%$, Soycake $30 \%$, Oat bran 5\%), PSRO (Plantain 60\%, Soycake $30 \%$, Rice bran 5\%, Oat bran 5\%). Cerolina (wheat and soybean produced by More Foods Lagos, Nigeria) and 100\% Plantain flour were used as control.

\section{Preparation of formulated food sample aqueous extracts} The aqueous extract of blended food sample was produced by dispersing each of the flour samples $(500 \mathrm{~g})$ into distilled water $(2.5 \mathrm{~L})$ and exhaustively marcerated for $12 \mathrm{~h}$. After marceration, the mixture was filtered using muslin cloth and Whatman No.1 filter paper; and the filtrate was concentrated using rotary evaporator (Model $349 / 2$, Corning Limited, England) at $40^{\circ} \mathrm{C}$ for $24 \mathrm{~h}$, and thereafter, the filtrate was freeze-dried to obtain food sample extract. The freeze-dried flour sample was packed in a plastic container, sealed and stored at room temperature $\left(\sim 27^{\circ} \mathrm{C}\right)$ until required for use.

\section{Determination of chemical composition Proximate composition}

Moisture, crude protein, fat, fiber and ash content of the food samples were determined according to AOAC methods [40]. Total carbohydrate content was calculated by subtracting the total percentage of moisture, fat, protein, fiber and ash from 100.

\section{Mineral content}

The minerals, i.e., calcium, magnesium, iron, zinc, copper and manganese, were determined using Atomic Absorption Spectrophotometer (AAS Model SP9). Sodium and potassium content were determined using flame photometer (Sherwood, U.K.) using $\mathrm{NaCl}$ and $\mathrm{KCl}$ as the standard. Phosphorus was determined by the vanado-molybdate colorimetric method of AOAC [40].

\section{Amino acid profile}

The amino acid composition of the food samples were determined using Automated Amino Acid Analyzer
(Model 6300; Beckman Coulter Inc., Fullerton, Calif., USA) as described by Mansouri et al. [41] and Hartcamp et al. [42].

\section{Quantitative determination of phytochemical constituents of the formulated food samples}

Tannins content of the food sample was determined as described by the method of Fagbemi et al. [43] with slight modification. Each of the sample extract was measured $(1.0 \mathrm{~g})$ and dispersed in distilled water $(10 \mathrm{ml})$, vortex vigorously and centrifuged at $3000 \times \mathrm{g}$ for $10 \mathrm{~min}$. The filtrate $(2.5 \mathrm{ml})$ and standard tannic acid solution $(2.5 \mathrm{ml})$ were separately dispersed into $50 \mathrm{ml}$ flask, respectively. Into each of the flask, folin-denis reagent (1.0 $\mathrm{ml}$ ) and saturated $\mathrm{Na}_{2} \mathrm{CO}_{3}$ solution $(2.5 \mathrm{ml})$ were poured into volumetric flask, respectively. Thereafter, the mixture was diluted with distilled water to mark in the volumetric flask $(50 \mathrm{ml})$ and incubated for $90 \mathrm{~min}$. at room temperature. The absorbance was measured at $250 \mathrm{~nm}$ in an electronic spectrophotometer (Genway model 6000i). Readings were taken with the reagent blank at zero. The tannin content was calculated.

$$
\text { Tannin }(\mathrm{mg} / \mathrm{g})=\frac{\text { Ab } x \text { Conc. } \mathrm{x} \text { Vf }}{\text { Ast } \mathrm{x} \text { Wt of sample } \mathrm{x} \text { Vol. }}
$$

Where

$\mathrm{Ab}=$ Absorbance of test sample, Ast $=$ Absorbance of standard solution, Conc. $=$ Concentration of standard solution, $\mathrm{W}=$ Weight of sample used $\mathrm{Vf}=$ Total volume of extract Vol. = Volume of extract.

Flavonoid was determined by the method reported by Boham and Kocipai [44]. The flour sample $(5.0 \mathrm{~g})$ was mixed with $50 \mathrm{~mL}$ of $80 \%$ aqueous methanol in a $250 \mathrm{ml}$ beaker, covered, and incubated for $24 \mathrm{~h}$ at room temperature. The mixture was filtered and the supernatant was discarded. The residue was re-extracted (thrice) with $50 \mathrm{ml}$ of ethanol. The combined mixture was filtered with Whatman filter paper number 42 (125 $\mathrm{mm})$, and the filtrate was transferred into a crucible. The filtrate was evaporated to dryness using a water bath, cooled in a desiccator and weighed until constant weight was obtained. The flavonoid was calculated.

Saponin was determined as described by the method of Obadoni and Ochuko [45]. Each of the flour samples $(20 \mathrm{~g})$ was poured into a $250 \mathrm{ml}$ conical flask conical flask containing $100 \mathrm{ml}$ of $20 \%$ aqueous ethanol. The mixture was transferred into a hot water bath maintained at $50{ }^{\circ} \mathrm{C}$ for $4 \mathrm{~h}$ with continuous stirring. The residue of the mixture was again extracted with another $100 \mathrm{ml}$ of $20 \%$ aqueous ethanol after filtration. Thereafter, the combined mixture was filtered and concentrated in a water bath at $90^{\circ} \mathrm{C}$. To the concentrated filtrate, $20 \mathrm{ml}$ diethyl ether was added and mixed 
vigorously to separate the aqueous layer from the ether layer, which was discarded. This purification process was repeated twice. The separated aqueous solution was mixed with $60 \mathrm{ml}$ of n-butanol. The mixture was then washed twice with $10 \mathrm{ml}$ of $5 \%$ aqueous sodium chloride, and the sodium chloride layer was separated and discarded, while the left over was re-concentrated in a water bath at $90{ }^{\circ} \mathrm{C}$ for $30 \mathrm{~min}$. The filtrate was then transferred into a crucible, and thereafter oven dried in hot-air oven (Gallenkamp, England) at $60^{\circ} \mathrm{C}$ to a constant weight. The saponin content was calculated.

Oxalate was determined as described by the modified method of Adeniyi et al. [46]. The sample $(2.5 \mathrm{~g})$ was digested with $10 \mathrm{ml} 6 \mathrm{M} \mathrm{HCl}$ at a temperature of $60^{\circ} \mathrm{C}$ for $60 \mathrm{~min}$. With constant stirring using a magnetic stirrer, and then filtered. To $5.0 \mathrm{ml}$ of the filtrate, $1.0 \mathrm{ml}$ of $5 \mathrm{M}$ ammonium hydroxide solution was added (to adjust $\mathrm{pH}$ ) until the colour of the solution changed from salmon pink colour to a faint yellow colour. Thereafter, to the solution, phenolphthalein indicator ( 2 drops), glacial acetic acid (3 drops) and 5\% calcium chloride $(5.0 \mathrm{ml})$ were added to precipitate insoluble oxalate, and the solution was allowed to incubate for $120 \mathrm{~min}$. at room temperature before centrifuged at $2500 \times \mathrm{g} 15 \mathrm{~min}$. The precipitate was washed with distilled hot water, $5.0 \mathrm{ml}$ of $3 \mathrm{M}$ tetraoxosulphate (VI) acid was added and warmed in a water bath at $60^{\circ} \mathrm{C}$ for $15 \mathrm{~min}$. Freshly prepared $0.01 \mathrm{M}$ potassium permanganate $\left(\mathrm{KMnO}_{4}\right)$ was titrated against $12.5 \mathrm{ml}$ of the filtrate until a faint pink colour, which persisted for about $30 \mathrm{~s}$. and the volume of $\mathrm{KMnO}_{4}$ used was read from the burette reading. The oxalate content was calculated $(\mathrm{mg} / \mathrm{g})$.

$$
\text { Oxalate }\left(\frac{\mathrm{mg}}{\mathrm{g}}\right)=V_{\mathrm{T}} \mathrm{x} 0.9004
$$

Where, $\mathrm{VT}=$ Titre volume $(\mathrm{ml})$.

Trypsin inhibition activity (TIA) was assayed described by the modified method of Smith et al. [47]. TIA was assayed in terms of the extent to which the extract of the defatted flour samples inhibited the action of bovine trypsin (EC 3.4.21.4) on the substrate benzoyl-DLarginine-p-nitrianilide (BAPNA) hydrochloric. The food sample $(1.0 \mathrm{~g})$ was extracted with $50 \mathrm{ml}, 10 \mathrm{mM} \mathrm{NaOH}$ at ambient temperature for $2 \mathrm{~h}$. with continuously shaking using a mechanical shaker (GallenKamp orbital shaken Surrey, UL). To the obtained slurry, $1 \mathrm{M} \mathrm{NaOH}$ was added to adjust the $\mathrm{pH}$ to 9.4. The suspension was diluted to $50 \mathrm{ml}$ with phosphate buffer, and into the test tube, inhibitor extract $(1.0 \mathrm{ml})$, trypsin solution $(1.0 \mathrm{ml})$ and $5 \%$ trichloroacetic acid $(6.0 \mathrm{ml})$ were added; and the mixture was incubated at room temperature for $60 \mathrm{~min}$. Thereafter, the suspension was filtered, and the absorbance of the filtrate and the blank $(6 \mathrm{ml}$ of $5 \%$ TCA and
$4 \mathrm{ml}$ buffer) were measured at $280 \mathrm{~nm}$ using spectrophotometer (Spectronic 601 model, Milton Roy Company, USA). Trypsin inhibitor activity was calculated in terms of mg pure trypsin (Sigma type lll, lot 20H0868).

$$
\mathrm{TIA}=\frac{2.632 \mathrm{DA} \mathrm{mg} \text { pure trypsin inhibited g-1 sample }}{\text { Weight of Sample }}
$$

Where $\mathrm{D}$ is the dilution factor, $\mathrm{A}$ is the change in absorbance at $410 \mathrm{~nm}$ due to trypsin inhibition per ml diluted sample extract.

The phenol in the food samples was determined as described by the method of George' et al. [48] with minor modifications. The food sample $(2.0 \mathrm{~g})$ was defatted using $100 \mathrm{ml}$ ether in a soxhlet apparatus for $90 \mathrm{~min}$. The defatted food sample $(1.0 \mathrm{~g})$ was dispersed in $50 \mathrm{~cm}^{3}$ boiled ether for $15 \mathrm{~min}$. to extract the phenolic components. Into the extracted filtrate $(5.0 \mathrm{ml})$, distilled water $(10.0 \mathrm{ml}), 0.1 \mathrm{~N}$ ammonium hydroxide solution $(2.0 \mathrm{ml})$, and concentrated amyl alcohol $(5.0 \mathrm{ml})$ were added, and the mixture was left to react for $25 \mathrm{~min}$. For colour development. The optical density was measured at the absorbance of $505 \mathrm{~nm}$. For the preparation of phenol standard curve, $0.20 \mathrm{~g}$ of tannic acid was dissolving in distilled water and diluted to $200 \mathrm{~mL}$ mark $(1 \mathrm{mg} / \mathrm{ml})$. The standard tannin acid solution was measured into five different test tube by varying its concentration between $0.2-1.0 \mathrm{mg} / \mathrm{ml}$. The standard tannic acid solution was then pipetted against the solution of $\mathrm{NH}_{3} \mathrm{OH}(2.0$ $\mathrm{ml})$, amyl alcohol $(5.0 \mathrm{ml})$, and distilled water $(10.0 \mathrm{ml})$. The solution was thereafter made up to $100 \mathrm{ml}$ volume and left to react for $25 \mathrm{~min}$. For colour development. The optical density was measured with spectrophotometer (Cecil 3021 spectrophotometer, Cambridge, United Kingdom) at $505 \mathrm{~nm}$. The amount of phenolic compounds was expressed as $\mathrm{mg}$ of gallic acid per $\mathrm{g}$ of extract (mgGAE/g).

The polyphenolic profiles were determined as described by Kelley et. al. [49] and Provan et al. [50]. The homogenised freeze-dried formulated food samples $(0.5 \mathrm{~g})$ were extracted with $25 \mathrm{ml}$ distilled water using homogeniser (Ultra Turrax T25 (IKA.Werke, Janke \& Kunkel). The aliquot of the aqueous food sample extract $(0.1 \mathrm{ml})$ was poured into GC vials and $50 \mu \mathrm{L}$ of an internal standard was added (3-[4-hydroxyphenyl]-1-propanol solution, $19.2 \mu \mathrm{g} / \mathrm{ml}$ ). Thereafter, the sample was evaporated to dryness under nitrogen and derivatised by addition of $250 \mu \mathrm{L} \mathrm{N}, O$-bis (trimethylsilyl) trifluoroacetamide (BSTFA) at $60^{\circ} \mathrm{C}$ for $15 \mathrm{~min}$. Analysis of the samples was performed by an Agilent (Wallborn, Germany) HP series GC 6890 N coupled with a HP 5973 MS detector (EI, $70 \mathrm{eV}$ ), a split-splitless injector, and an HP 7683 autosampler in an HP-5 MS capillary column (5\% phenyl-95\% methyl siloxane, $30 \mathrm{~m} \times 0.25 \mathrm{~mm} \times$ 
$250 \mu \mathrm{m})$, and 3-(4-hydroxyphenyl)-1-propanol was used as the standard.

\section{Determination of functional and pasting properties}

The functional properties of flour blends, that is, bulk density, water absorption, oil absorption, foaming capacity/stability, emulsion capacity/stability and gelatinization were determined by the methods described by Iwe et al. [51]. Swelling capacity was determined according to method described by Akpata and Miachi [52]. Pasting properties of the flour blends were determined using the Rapid Visco Analyzer (RVA Model 3c, Newport Scientific PTY Ltd., Sydney) as described by AACC [53].

\section{In-vitro antioxidant assay of the formulated food sample aqueous extracts}

The in-vitro antioxidant assay of the aqueous extracts of the food sample using standard methods. The free radical scavenging activity of the food samples was determined against 2,2-azino-bis (3-ethylbenzthiazoline-6sulphonate (ABTS) as described by Re et al. [54]. The 2,2- diphenyl-1-picryhydrazyl (DPPH) free radical scavenging was determined as described by Aluko and Monu [55]. Metal chelating activity of the formulated food aqueous extract was determined using the method of Xie et al. [56]. Ferric reducing antioxidant activity (FRAP) was determined according to Zhang and Lin [57]. The hydroxyl free radical scavenging activity of the food extract was determined according to the method reported by Girgih et al. [58].

Calculation: The dietary antioxidant activity index (DAAI) were calculated for each of the formulated food samples as follows:

$$
D A A I=\left(\frac{\text { Final concentration of antioxidant eg DPPH }(\mathrm{mg} / \mathrm{dL}}{I C_{50}(\mathrm{mg} / \mathrm{dL})}\right)
$$

Low antioxidant activity DAAI $<0.5$, moderate antioxidant activity DAAI $=0.5-1.0$, strong antioxidant activity DAAI $=1.0-2.0$, very strong $=$ DAAI $>2.0[59]$.

\section{Experimental design and determination of blood lipid profile in rats \\ Ethical approval}

The experiments on animals were conducted in accordance with the force laws and regulations as regards animal use and care as contained in the Canadian Council on Animal Care Guidelines and Protocol Review [60].

\section{Experimental design}

Forty male albino rats (190-200 g) were purchased from the Department of Biochemistry, Federal University of Technology, Akure, Nigeria. The rats were divided into seven groups $(n=5)$; and were housed in individual stainless steel cages maintained at standard environmental condition of controlled temperature $24 \pm 5{ }^{\circ} \mathrm{C}$, illumination (12 h light/12 h dark cycles), relative humidity of $60 \pm 4 \%$ for 7 -day acclimatization.

\section{Formulation and feeding of high fat diet to the experimental rats}

After 7-day acclimatization, the experimental rats were fed on high-fat diets (HFD) for diet-induced obesity for 28 days. The high-fat diets $(\mathrm{g} / 100 \mathrm{~g})$ were formulated from the blending of skimmed milk $(50 \mathrm{~g})$, lard $(30 \mathrm{~g})$, cellulose $(9 \mathrm{~g})$, corn starch $(7 \mathrm{~g})$ and premix $(4 \mathrm{~g})$ as described by Kim et al. [61] and Vatandoust et al. [62] with slight modification. The HFD ( $20 \mathrm{~g} /$ day $)$ and water were provided to the rats' ad libitum throughout the experimental period.

\section{Grouping and feeding of experimental diets to the rats}

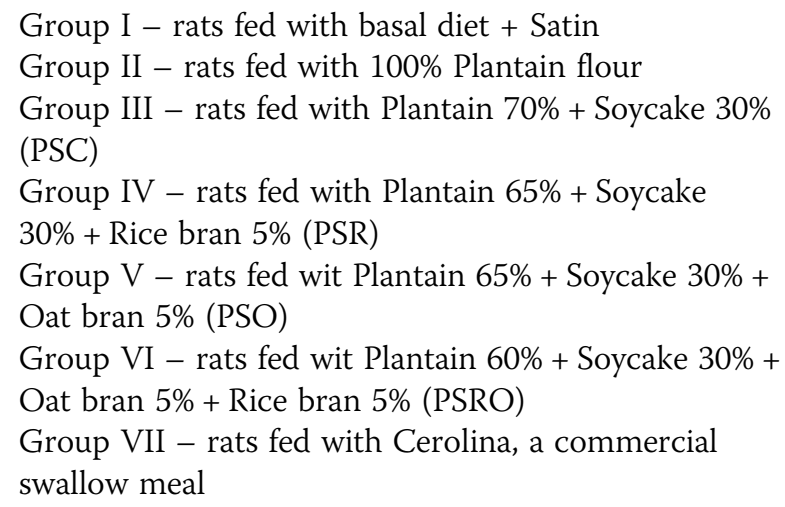

The rats were administered with the experimental diets and water ad libitum; while Satin (an anti-cholesterol agent $=10 \mathrm{mg} / \mathrm{kg}$ body weight) was administered orally to the rats for 28 days.

\section{Determination of blood lipid profile}

At the end of 28 days, the rats were sacrificed after a 12h overnight fast, and blood sample was collected via cardiac puncture into clean test tube. Total Cholesterol (TC) was determined by the method described by Siedel et al. [63], Triglyceride concentration (TG) by the method described by Tietz [64], and HDL-cholesterol was determined using the method reported by Jacobs et al. [65]. The serum High-density lipoprotein concentration (HDL-conc.) was determined by the dextran sulphatemagnesium (II) precipitation method reported by Albers et al. [66]. Low-Density Lipoprotein (LDL-concentration), Very Low-Density Lipoprotein (VLDL-conc.), Atherogenic Index (AI) and Coronary Risk Index (CRI) were calculated: 
Low-density lipoprotein (LDL)

The Low Density Lipoprotein was calculated according to the method described by Friedewald et al. [67].

$$
\mathrm{LDL}=\frac{\text { Total cholesterol }- \text { Triglycerides }-\mathrm{HDL}}{5}
$$

Very Low-Density Lipoprotein cholesterol (VLDL-

C) [68].

VLDL-C $(\mathrm{mg} / \mathrm{dL})=$ Triglycerides / 2.2.

Atherogenic index (AI) [69].

$$
\mathrm{AI}=\frac{\mathrm{LDL}-\text { cholesterol }}{\text { HDL-cholesterol }}
$$

\section{Coronary Risk Index (CRI) [70].}

$$
\mathrm{CRI}=\frac{\text { Total cholesterol }}{\text { HDL-cholesterol }}
$$

Where,

$V L D L-C$ is very low-density lipoprotein cholesterol.

$L D L-C$ is low-density lipoprotein cholesterol.

$H D L-C$ is high-density lipoprotein cholesterol.

\section{Data analysis}

Data in triplicate readings were analysed and results presented in mean \pm standard error mean (SEM). One-way Analysis of Variance (ANOVA) was used to analyze the means. Significant differences between means were determined using Duncan with level of significant at $p \leq$ 0.05 .

\section{Results}

\section{Nutritional composition}

The nutrient composition of the blended flour samples is presented in Table 1. The moisture content, total fiber, crude protein and energy value of the food samples ranged from 5 to $5.5 \mathrm{~g} / 100 \mathrm{~g}, 2.2-4.97 \mathrm{~g} / 100 \mathrm{~g}, 16.44-$ $19.59 \mathrm{~g} / 100 \mathrm{~g}$ and $369.7-385.5 \mathrm{kcal} / 100 \mathrm{~g}$, respectively.

The mineral composition of the food products showed that phosphorous $(24.37-33.36 \mathrm{mg} / 100 \mathrm{~g})$ was the most abundant, while copper $(0.12-0.16 \mathrm{mg} / 100 \mathrm{~g})$ had the least concentration in the food products, and the values were comparatively higher than in $100 \%$ plantain flour $(7.43,0.09 \mathrm{mg} / 100 \mathrm{~g})$ and cerolina $(17.31,0.09 \mathrm{mg} / 100 \mathrm{~g})$. $\mathrm{The} \mathrm{Na} / \mathrm{K}$ and $\mathrm{Ca} / \mathrm{P}$ molar ratios were lower than critical values of $<1,>1$, respectively. The amino acid compositions and predicted nutritional quality of the food products are presented in Tables $2 \& 3$, respectively. The

\begin{tabular}{|c|c|c|c|c|c|c|c|}
\hline Samples & $100 \%$ Plantain & PSC & PSR & PSO & PSRO & Cerolina & *Ref. \\
\hline Moisture & $5.10 \pm 0.04^{d}$ & $5.20 \pm 0.02^{c}$ & $5.10 \pm 0.03^{d}$ & $5.30 \pm 0.02^{b}$ & $5.50 \pm 0.05^{\mathrm{a}}$ & $5.60 \pm 0.04^{a}$ & $<10$ \\
\hline Ash & $2.00 \pm 0.02^{e}$ & $3.50 \pm 0.01^{c}$ & $4.40 \pm 0.05^{\mathrm{a}}$ & $3.00 \pm 0.06^{d}$ & $4.00 \pm 0.03^{b}$ & $2.00 \pm 0.04^{e}$ & $<3$ \\
\hline Fat & $2.00 \pm 0.02^{f}$ & $5.00 \pm 0.01^{\mathrm{e}}$ & $6.09 \pm 0.03^{d}$ & $6.29 \pm 0.05^{c}$ & $6.87 \pm 0.03^{b}$ & $8.67 \pm 0.04^{\mathrm{a}}$ & $10-25$ \\
\hline Fibre & $1.33 \pm 0.02^{f}$ & $2.20 \pm 0.04^{\mathrm{e}}$ & $5.69 \pm 0.03^{a}$ & $3.19 \pm 0.04^{d}$ & $4.97 \pm 0.09^{b}$ & $3.55 \pm 0.06^{c}$ & $<5$ \\
\hline Protein & $5.69 \pm 0.09^{f}$ & $16.44 \pm 0.04^{e}$ & $16.52 \pm 0.06^{d}$ & $19.43 \pm 0.07^{b}$ & $19.59 \pm 0.04^{\mathrm{a}}$ & $18.01 \pm 0.05^{c}$ & $>16$ \\
\hline Carbohydrates & $83.88 \pm 0.09^{a}$ & $67.66 \pm 0.04^{b}$ & $62.20 \pm 0.05^{c}$ & $62.79 \pm 0.07^{c}$ & $59.07 \pm 0.03^{d}$ & $62.17 \pm 0.08^{c}$ & 64 \\
\hline Energy & $376.28 \pm 0.17^{d}$ & $381.40 \pm 0.21^{c}$ & $369.69 \pm 0.15^{\mathrm{e}}$ & $385.49 \pm 0.23^{b}$ & $376.47 \pm 0.10^{d}$ & $398.75 \pm 0.24^{a}$ & $400-425$ \\
\hline \multicolumn{8}{|l|}{ Minerals } \\
\hline $\mathrm{Ca}$ & $2.32 \pm 0.00^{d}$ & $2.41 \pm 0.01^{c}$ & $2.24 \pm 0.01^{\mathrm{e}}$ & $3.27 \pm 0.00^{b}$ & $4.33 \pm 0.01^{\mathrm{a}}$ & $2.38 \pm 0.01^{c}$ & 500 \\
\hline $\mathrm{Mg}$ & $2.47 \pm 0.00^{\mathrm{e}}$ & $2.54 \pm 0.01^{d}$ & $2.67 \pm 0.01^{c}$ & $2.85 \pm 0.01^{b}$ & $3.54 \pm 0.01^{\mathrm{a}}$ & $2.40 \pm 0.01^{f}$ & 76 \\
\hline P & $7.43 \pm 0.01^{f}$ & $24.37 \pm 0.01^{d}$ & $26.27 \pm 0.01^{c}$ & $33.36 \pm 0.00^{a}$ & $29.35 \pm 0.01^{b}$ & $17.31 \pm 0.00^{e}$ & 456 \\
\hline K & $8.51 \pm 0.01^{e}$ & $9.15 \pm 0.01^{d}$ & $9.64 \pm 0.01^{c}$ & $10.12 \pm 0.01^{b}$ & $14.82 \pm 0.01^{\mathrm{a}}$ & $7.38 \pm 0.00^{f}$ & 516 \\
\hline $\mathrm{Na}$ & $4.27 \pm 0.00^{d}$ & $4.53 \pm 0.01^{c}$ & $4.59 \pm 0.01^{b}$ & $4.57 \pm 0.00^{b}$ & $4.66 \pm 0.01^{\mathrm{a}}$ & $3.71 \pm 0.00^{e}$ & 296 \\
\hline $\mathrm{Fe}$ & $2.16 \pm 0.00^{e}$ & $2.34 \pm 0.01^{d}$ & $2.83 \pm 0.01^{b}$ & $2.83 \pm 0.00^{b}$ & $2.95 \pm 0.01^{\mathrm{a}}$ & $2.53 \pm 0.01^{c}$ & 16 \\
\hline $\mathrm{Zn}$ & $2.64 \pm 0.00^{c}$ & $1.53 \pm 0.01^{f}$ & $2.38 \pm 0.01^{e}$ & $2.74 \pm 0.01^{b}$ & $2.84 \pm 0.01^{\mathrm{a}}$ & $2.56 \pm 0.01^{d}$ & 3.20 \\
\hline $\mathrm{Cu}$ & $0.09 \pm 0.01^{b c}$ & $0.12 \pm 0.00^{b}$ & $0.10 \pm 0.01^{b}$ & $0.15 \pm 0.01^{a}$ & $0.16 \pm 0.01^{\mathrm{a}}$ & $0.09 \pm 0.01^{b c}$ & 0.89 \\
\hline $\mathrm{Mn}$ & $0.19 \pm 0.00^{d}$ & $0.24 \pm 0.00^{c}$ & $0.31 \pm 0.01^{a}$ & $0.27 \pm 0.01^{b}$ & $0.29 \pm 0.01^{\mathrm{ab}}$ & $0.16 \pm 0.01^{e}$ & 1.50 \\
\hline $\mathrm{Na} / \mathrm{K}$ & $0.50^{\mathrm{a}}$ & $0.49^{a}$ & $0.48^{\mathrm{a}}$ & $0.45^{\mathrm{b}}$ & $0.31^{c}$ & $0.50^{\mathrm{a}}$ & $<1.00$ \\
\hline $\mathrm{Ca} / \mathrm{P}$ & $0.31^{\mathrm{a}}$ & $0.10^{c}$ & $0.09^{c}$ & $0.10^{c}$ & $0.15^{\mathrm{b}}$ & $0.09^{c}$ & $>1.00$ \\
\hline
\end{tabular}

Table 1 Proximate composition (g/100 g DM), energy value (Kcal/100 g) and minerals (mg/100 g) of plantain, soycake, rice-bran and oat-bran flour blends and control samples

Means $( \pm$ SEM) with different alphabetical superscripts in the same column are significantly different at $P<0.05$ Key: 100\% Plantain flour; PSC: Plantain: Soycake (70:30\%); PSR: Plantain: Soycake: Rice bran (65:30:5\%); PSO: Plantain: Soycake: Oat bran (65:30:5\%); PSRO: Plantain: Soycake: Rice bran: Oat bran (60:30:5:5); Cerolina: a commercial flour meal. Ref: NIS [118] 
Table 2 Amino acid profile (g/100 g protein) of plantain, soycake, rice bran and oat bran flour and control sample

\begin{tabular}{|c|c|c|c|c|c|c|c|c|}
\hline Amino Acids & $\begin{array}{l}100 \% \\
\text { Plantain }\end{array}$ & PCS & PSR & PSO & PSRO & Cerolina & ${ }^{*}$ Adult & ${ }^{*}$ Children \\
\hline \multicolumn{9}{|c|}{ Non Essential Amino Acids (NEAAs) } \\
\hline Glycine & $3.97^{\mathrm{d}}$ & $4.49^{c}$ & $4.48^{c}$ & $4.48^{\mathrm{c}}$ & $4.62^{\mathrm{b}}$ & $4.88^{\mathrm{a}}$ & - & - \\
\hline Alanine & $3.73^{f}$ & $4.46^{\mathrm{d}}$ & $4.48^{\mathrm{c}}$ & $4.44^{\mathrm{e}}$ & $4.50^{\mathrm{b}}$ & $5.14^{\mathrm{a}}$ & - & - \\
\hline Serine & $5.24^{f}$ & $5.51^{b}$ & $5.33^{\mathrm{e}}$ & $5.48^{\mathrm{c}}$ & $5.45^{\mathrm{d}}$ & $5.66^{\mathrm{a}}$ & - & - \\
\hline Proline & $7.45^{\mathrm{a}}$ & $5.05^{\mathrm{d}}$ & $5.07^{c}$ & $5.09^{\mathrm{b}}$ & $5.10^{\mathrm{b}}$ & $4.04^{\mathrm{e}}$ & - & - \\
\hline Aspartic & $9.44^{f}$ & $12.40^{\mathrm{b}}$ & $12.36^{\mathrm{c}}$ & $11.96^{\mathrm{e}}$ & $12.07^{\mathrm{d}}$ & $14.91^{\mathrm{a}}$ & - & - \\
\hline Cysteine & $1.59^{\mathrm{a}}$ & $1.07^{\mathrm{f}}$ & $1.16^{\mathrm{e}}$ & $1.32^{\mathrm{c}}$ & $1.21^{\mathrm{d}}$ & $1.35^{\mathrm{b}}$ & - & - \\
\hline Glutamic & $24.32^{\mathrm{a}}$ & $17.94^{\mathrm{d}}$ & $17.87^{\mathrm{e}}$ & $18.28^{b}$ & $18.15^{c}$ & $15.13^{f}$ & - & - \\
\hline Tyrosine & $2.80^{\mathrm{e}}$ & $3.27^{d}$ & $3.41^{\mathrm{c}}$ & $3.46^{\mathrm{a}}$ & $3.28^{\mathrm{d}}$ & $3.44^{\mathrm{b}}$ & - & - \\
\hline Arginine & $5.91^{f}$ & $7.23^{\mathrm{b}}$ & $7.18^{c}$ & $7.16^{\mathrm{d}}$ & $7.05^{e}$ & $8.18^{\mathrm{a}}$ & - & - \\
\hline ¿NEAAs & $65.13^{\mathrm{a}}$ & $62.25^{c}$ & $61.99^{\mathrm{e}}$ & $62.22^{\mathrm{d}}$ & $62.26^{c}$ & $62.94^{\mathrm{b}}$ & - & - \\
\hline \multicolumn{9}{|c|}{ Essential Amino Acids (EAAs) } \\
\hline Phenylalanine & $5.12^{c}$ & $5.10^{d}$ & $5.17^{\mathrm{b}}$ & $5.18^{\mathrm{b}}$ & $5.22^{\mathrm{a}}$ & $4.62^{\mathrm{e}}$ & 2.50 & 6.90 \\
\hline Histidine & $1.93^{\mathrm{e}}$ & $2.46^{\mathrm{b}}$ & $2.43^{c}$ & $2.40^{\mathrm{d}}$ & $2.41^{\mathrm{d}}$ & $3.97^{\mathrm{a}}$ & - & 1.00 \\
\hline Methionine & $1.52^{\mathrm{b}}$ & $1.26^{f}$ & $1.41^{\mathrm{d}}$ & $1.47^{\mathrm{c}}$ & $1.37^{e}$ & $1.63^{\mathrm{a}}$ & 1.50 & 2.70 \\
\hline Valine & $4.55^{\mathrm{e}}$ & $5.00^{b}$ & $4.97^{\mathrm{d}}$ & $4.97^{\mathrm{d}}$ & $5.06^{\mathrm{a}}$ & $4.99^{c}$ & 2.60 & 3.80 \\
\hline Tryptophan & $1.22^{\mathrm{d}}$ & $1.33^{\mathrm{b}}$ & $1.31^{\mathrm{c}}$ & $1.37^{\mathrm{a}}$ & $1.23^{\mathrm{e}}$ & $1.22^{\mathrm{e}}$ & 0.40 & 1.25 \\
\hline Threonine & $3.48^{f}$ & $4.10^{\mathrm{b}}$ & $4.07^{c}$ & $4.01^{\mathrm{d}}$ & $4.13^{\mathrm{a}}$ & $3.66^{\mathrm{e}}$ & 1.50 & 3.70 \\
\hline Isoleucine & $4.19^{c}$ & $4.50^{\mathrm{b}}$ & $4.49^{b}$ & $4.49^{b}$ & $4.53^{\mathrm{a}}$ & $3.53^{\mathrm{d}}$ & 2.00 & 3.10 \\
\hline Leucine & $7.34^{d}$ & $7.73^{\mathrm{a}}$ & $7.72^{\mathrm{a}}$ & $7.64^{c}$ & $7.70^{\mathrm{b}}$ & $6.61^{e}$ & 3.90 & 7.30 \\
\hline Lysine & $4.76^{\mathrm{f}}$ & $6.05^{\mathrm{a}}$ & $6.02^{\mathrm{b}}$ & $5.78^{\mathrm{d}}$ & $5.85^{c}$ & $5.67^{\mathrm{e}}$ & 3.00 & 6.40 \\
\hline ¿EAAs & $34.87^{\mathrm{e}}$ & $37.75^{c}$ & $38.01^{\mathrm{a}}$ & $37.78^{\mathrm{b}}$ & $37.74^{c}$ & $37.06^{d}$ & - & - \\
\hline TAAs & 100.00 & 100.00 & 100.00 & 100.00 & 100.00 & 100.00 & & \\
\hline
\end{tabular}

Key: 100\% Plantain flour; PSC: Plantain: Soycake (70:30\%); PSR: Plantain: Soycake: Rice bran (65:30:5\%); PSO: Plantain: Soycake: Oat bran (65:30:5\%); PSRO: Plantain: Soycake: Rice bran: Oat bran (60:30:5:5); Cerolina: a commercial flour meal; NEAAs Non-essential amino acids, EAAs Essential amino acids, TAAs Total amino acids ${ }^{*}$ RDA of essential amino acids ((mg/100 gb.w)) for Adult and Children $(<5$ yrs.) $[119,120]$

Table 3 Predicted nutritional qualities of plantain, soycake, rice bran and oat bran flour and control sample

\begin{tabular}{|c|c|c|c|c|c|c|}
\hline Parameters & $100 \%$ Plantain & PCS & PSR & PSO & PSRO & Cerolina \\
\hline HAAs (g/100 g protein) & $37.52^{d}$ & $38.76^{c}$ & $39.18^{b}$ & $39.43^{a}$ & $39.19^{b}$ & $36.55^{e}$ \\
\hline PCAAs(g/100 g protein) & $6.69^{f}$ & $8.51^{\mathrm{b}}$ & $8.45^{\mathrm{c}}$ & $8.18^{e}$ & $8.26^{d}$ & $9.64^{a}$ \\
\hline NCAAs(g/100 g protein) & $33.76^{\mathrm{a}}$ & $30.33^{b}$ & $30.22^{d}$ & $30.24^{c}$ & $30.22^{d}$ & $30.04^{f}$ \\
\hline ArAAs (g/100 g protein) & $9.15^{f}$ & $9.69^{d}$ & $9.89^{b}$ & $10.01^{\mathrm{a}}$ & $9.74^{c}$ & $9.27^{e}$ \\
\hline SCAA (g/100 g protein) & $3.10^{\mathrm{a}}$ & $2.33^{e}$ & $2.58^{\mathrm{d}}$ & $2.79^{c}$ & $2.57^{d}$ & $2.98^{\mathrm{b}}$ \\
\hline BCAA (g/100 g protein) & $16.09^{d}$ & $17.23^{b}$ & $17.18^{c}$ & $17.11^{c}$ & $17.28^{\mathrm{a}}$ & $15.13^{e}$ \\
\hline TEAA/TNEAA & $0.54^{\mathrm{b}}$ & $0.61^{\mathrm{a}}$ & $0.61^{\mathrm{a}}$ & $0.61^{\mathrm{a}}$ & $0.61^{\mathrm{a}}$ & $0.59^{\mathrm{a}}$ \\
\hline PER $(\mathrm{g} / 100 \mathrm{~g})$ & $2.42^{d}$ & $2.68^{\mathrm{a}}$ & $2.22^{\mathrm{e}}$ & $2.58^{c}$ & $2.67^{\mathrm{a}}$ & $2.63^{b}$ \\
\hline EAAI (\%) & $62.84^{f}$ & $67.79^{\mathrm{d}}$ & $68.31^{c}$ & $68.38^{\mathrm{b}}$ & $76.31^{a}$ & $67.59^{e}$ \\
\hline P-BV (\%) & $54.11^{f}$ & $62.19^{d}$ & $62.76^{c}$ & $62.83^{b}$ & $71.48^{\mathrm{a}}$ & $61.98^{\mathrm{e}}$ \\
\hline $\mathrm{NI}(\%)$ & $11.32^{d}$ & $11.14^{f}$ & $11.29^{\mathrm{e}}$ & $13.40^{c}$ & $14.83^{\mathrm{a}}$ & $3.85^{b}$ \\
\hline ARG/LYS & $1.24^{b}$ & $1.20^{c}$ & $1.19^{c}$ & $1.24^{b}$ & $1.20^{c}$ & $1.44^{\mathrm{a}}$ \\
\hline LAAs & Histidine & Methionine & Methionine & Methionine & Methionine & Methionine \\
\hline AAAs & Methionine & Arginine & Arginine & Arginine & Arginine & Arginine \\
\hline
\end{tabular}

Means ( \pm SEM) with different alphabetical superscripts in the same column are significantly different at $P<0.05$

Key: 100\% Plantain flour; PSC: Plantain: Soycake (70:30\%); PSR: Plantain: Soycake: Rice bran (65:30:5\%); PSO: Plantain: Soycake: Oat bran (65:30:5\%); PSRO: Plantain: Soycake: Rice bran: Oat bran (60:30:5:5); Cerolina: a commercial flour meal. HAAs Hydrophobic amino acids, PCAAs Positively charged amino acids, NCAAs Negatively charged amino acids, ArAAs Aromatic amino acids, SAAs Sulphur containing amino acids, BCAAs Branch chain amino acids, EAAs Essential amino acids, NEAAs Non-essential amino acids, ARG/LYS Arginine/ lysine ratio, PER Protein efficiency ratio, P-BV Predicted biological value, NI Nitrogen index, LAAs Limiting Amino Acids, AAAs Abundant amino acids 
essential amino acids profile, essential amino acid index (EAAI), predicted-biological values (P-BV) and nutritional index of the formulated food products ranged from $37.74-38.01$ (g/100 g protein), 68.31-76.31\%, $62.19-71.48 \%$ and $11.14-14.83 \%$, respectively, and were significantly $(p<0.05)$ higher than $100 \%$ plantain flour and cerolina. The branched chain amino acids (BCAAs), aromatic amino acids (ArAAs) and Arginine/lysine molar ratios of the food products ranged from 17.11$17.28 \mathrm{~g} / 100 \mathrm{~g}$ protein, $9.69-10.01 \mathrm{~g} / 100 \mathrm{~g}$ protein and $1.19-1.24$, respectively, and were higher than $100 \%$ plantain and cerolina, except in Arginine/Lysine ratio.

\section{Phytochemicals and phenolic compounds}

The phytochemical concentration $(\mathrm{mg} / \mathrm{g})$ in the formulated food samples showed that phenol, flavonoid and tannin varied from $11.35-18.42,0.30-0.54$ and $1.97-$ 3.09 , respectively; while phytate, oxalate and trypsin varied between 2.76-3.58, 0.54-0.72 and 5.12-10.14, respectively (Table 4). Comparatively, the concentration of these phytochemicals was lower than critical values, but were similar to that of $100 \%$ plantain flour and Cerolina.

The phenolic profile of the formulated food samples is presented in Table 5. The concentration of phenolic compounds $(\mathrm{mg} / \mathrm{g})$ in the formulated food samples were gallic acid (25.33-31.26), caffeic acid (2.75-4.61), Ferulic acid (5.16-12.73), luteolin (16.31-23.60), kaempferol (21.51-30.64), quercetin (24.28-37.13), chlorogenic acid (42.25-59.78), myricetin (28.41-38.41), 3,5-dicaffeoylquinic acid (27.17-41.59) and 4,5-dicaffeoylquinic acid (39.96-51.28). The PSRO had the highest phenolic concentration, while PSC had the lowest concentration; and the values were either higher or lower than in 100\% plantain and Cerolina samples.

\section{Functional, pasting and colour properties}

The functional property of plantain, soycake, rice-bran and oat-bran composite flour and control samples is presented in Table 6. Bulk density (BD) of the blended flour samples ranged from 0.67 to $0.71 \mathrm{~g} / \mathrm{mL}$, and was significantly $(p<0.05)$ lower than $100 \%$ plantain $(0.74 \mathrm{~g} /$
$\mathrm{mL})$ and cerolina $(0.73 \mathrm{~g} / \mathrm{mL})$. Water absorption capacity of the flour samples ranged from $0.67-0.71 \mathrm{~g} / \mathrm{mL}$, and was comparable to that of $100 \%$ plantain flour and Cerolina.

The least gelation concentration (LGC), that is, the lowest protein concentration at which gel remained in the inverted tube, ranged from 6 to $8 \%$, and the values were comparable to that of $100 \%$ plantain and Cerolina. However, lowest LGC value was observed in the products with rice-bran and oat-bran inclusion.

The peak viscosity (i.e., an index of the ability of starch-based foods to swell freely before their physical breakdown) values in this study ranged between 422 and $584 \mathrm{RVU}$, and the values were significantly lower than $100 \%$ plantain flour (2186 RVU) and cerolina (792 RVU).

The trough viscosity, i.e., the point at which the viscosity reaches its minimum during either heating or cooling processes, ranged from 362 to 500 for PSR and PSRO, respectively. These values were significantly lower than in 100\% plantain flour (2065 RVU) and Cerolina (571 RVU). The breakdown viscosity of the flour samples ranged from 60 to 84 RVU, while of $100 \%$ plantain flour and Cerolina were $121 \mathrm{RVU}$ and $221 \mathrm{RVU}$, respectively.

The values of final viscosity, setback and pasting temperature of the experimental flour samples ranged from 717 to $996 \mathrm{RVU}, 355-496 \mathrm{RVU}$ and 92-94 RVU, respectively, and were lower than in $100 \%$ plantain flour, but comparable to Cerolina. For the peak time, the values were $7 \mathrm{~min}$. and $5.27 \mathrm{~min}$. For the experimental flour samples, $100 \%$ plantain flour and cerolina, respectively.

\section{Antioxidant activities}

The antioxidant activity of the formulated food sample extracts is presented in Table 7. The antioxidant activities of the food samples against DPPH, ABTS, $\mathrm{Fe}^{2+}$ chelation, FRAP and $\mathrm{OH}$ free radical were concentration dependent, that is, as the concentration of the food sample extracts increased, the inhibitory activities of free

Table 4 Phytochemical composition (mg/g) of plantain, soycake, rice bran and oat bran flour and control samples

\begin{tabular}{llllllll}
\hline Parameters & $\mathbf{1 0 0} \%$ Plantain & PSC & PSR & PSO & PSRO & Cerolina & *CV \\
\hline Total Phenol & $16.18 \pm 0.03^{\mathrm{f}}$ & $19.08 \pm 0.03^{\mathrm{e}}$ & $24.28 \pm 0.04^{\mathrm{c}}$ & $25.97 \pm 0.03^{\mathrm{b}}$ & $26.15 \pm 0.03^{\mathrm{a}}$ & $21.58 \pm 0.04^{\mathrm{d}}$ & - \\
Flavonoid & $0.84 \pm 0.01^{\mathrm{d}}$ & $0.91 \pm 0.01^{\mathrm{c}}$ & $0.92 \pm 0.01^{\mathrm{c}}$ & $0.96 \pm 0.01^{\mathrm{b}}$ & $1.15 \pm 0.02^{\mathrm{a}}$ & $0.92 \pm 0.01^{\mathrm{c}}$ & - \\
Tannin & $5.19 \pm 0.01^{\mathrm{f}}$ & $5.65 \pm 0.02^{\mathrm{e}}$ & $5.97 \pm 0.01^{\mathrm{c}}$ & $6.04 \pm 0.02^{\mathrm{b}}$ & $6.58 \pm 0.01^{\mathrm{a}}$ & $5.79 \pm 0.03^{\mathrm{d}}$ & $3.0 \mathrm{mg} / 100 \mathrm{~g}$ \\
Phytate & $7.00 \pm 0.02^{\mathrm{a}}$ & $6.18 \pm 0.23^{\mathrm{b}}$ & $5.77 \pm 0.01^{\mathrm{b}}$ & $4.53 \pm 0.24^{\mathrm{c}}$ & $3.71 \pm 0.03^{\mathrm{d}}$ & $3.71 \pm 0.24^{\mathrm{d}}$ & $5-6 \mathrm{~g} / 100 \mathrm{~g}$ \\
Oxalate & $0.59 \pm 0.02^{\mathrm{a}}$ & $0.54 \pm 0.01^{\mathrm{a}}$ & $0.41 \pm 0.02^{\mathrm{b}}$ & $0.32 \pm 0.02^{\mathrm{c}}$ & $0.18 \pm 0.00^{\mathrm{d}}$ & $0.45 \pm 0.01^{\mathrm{b}}$ & - \\
Trypsin Inhibitor & $10.02 \pm 0.06^{\mathrm{d}}$ & $8.32 \pm 0.09^{\mathrm{f}}$ & $9.27 \pm 0.02^{\mathrm{e}}$ & $10.61 \pm 0.02^{\mathrm{c}}$ & $13.13 \pm 0.03^{\mathrm{b}}$ & $17.03 \pm 0.03^{\mathrm{a}}$ & $0.25 \mathrm{~g} / 100 \mathrm{~g}$ \\
\hline
\end{tabular}

Means $( \pm$ SEM) with different alphabetical superscripts in the same column are significantly different at $P<0.05$

Key: 100\% Plantain flour; PSC: Plantain: Soycake (70:30\%); PSR: Plantain: Soycake: Rice bran (65:30:5\%); PSO: Plantain: Soycake: Oat bran (65:30:5\%); PSRO: Plantain: Soycake: Rice bran: Oat bran (60:30:5:5); Cerolina: a commercial flour meal

${ }^{*}$ CV: phytate:calcium; phytate:zinc [121]; phytate:iron [122]; and phytate:calcium/zinc [123] 
Table 5 Phenolic profile (mg/g) of plantain, soycake, rice bran and oat bran dough meal and control samples

\begin{tabular}{|c|c|c|c|c|c|c|}
\hline Phenolic profile & $100 \%$ Plantain & PSC & PSR & PSO & PSRO & Cerolina \\
\hline Catechin & $29.54^{a}$ & $0.00^{b}$ & $0.00^{\mathrm{b}}$ & $0.00^{\mathrm{b}}$ & $0.00^{\mathrm{b}}$ & $0.00^{b}$ \\
\hline p-coumaric acid & nd & $0.34^{e}$ & $0.39^{d}$ & $0.46^{c}$ & $0.71^{\mathrm{a}}$ & $0.51^{b}$ \\
\hline Epicatechin & $4.30^{\mathrm{a}}$ & nd & nd & nd & nd & nd \\
\hline p-hydroxybenzoic acid & $5.92^{\mathrm{a}}$ & $0.02^{\mathrm{b}}$ & $0.02^{\mathrm{b}}$ & $0.02^{\mathrm{b}}$ & $0.02^{\mathrm{b}}$ & $0.02^{b}$ \\
\hline Gallic acid & $43.79^{a}$ & $25.33^{e}$ & $26.51^{d}$ & $29.80^{c}$ & $31.26^{\mathrm{b}}$ & $22.90^{f}$ \\
\hline Caffeic acid & $10.74^{a}$ & $2.75^{d}$ & $3.30^{c}$ & $4.61^{b}$ & $4.61^{b}$ & $2.55^{\mathrm{e}}$ \\
\hline Syringic acid & $5.26^{\mathrm{a}}$ & $0.02^{\mathrm{b}}$ & $0.02^{\mathrm{b}}$ & $0.02^{\mathrm{b}}$ & $0.01^{\mathrm{b}}$ & $0.01^{b}$ \\
\hline Ferulic acid & $3.40^{f}$ & $5.16^{d}$ & $6.43^{c}$ & $8.41^{\mathrm{b}}$ & $12.73^{\mathrm{a}}$ & $4.13^{\mathrm{e}}$ \\
\hline Apigenin & $5.90^{\mathrm{a}}$ & nd & nd & nd & nd & nd \\
\hline Luteolin & $18.39^{d}$ & $16.31^{f}$ & $18.50^{c}$ & $21.91^{b}$ & $23.60^{a}$ & $17.44^{e}$ \\
\hline Naringenin & $1.32^{\mathrm{a}}$ & $0.01^{b}$ & $0.01^{\mathrm{b}}$ & $0.01^{\mathrm{b}}$ & $0.01^{\mathrm{b}}$ & $0.01^{b}$ \\
\hline Kaempferol & $24.22^{c}$ & $21.51^{\mathrm{e}}$ & $23.27^{d}$ & $28.53^{b}$ & $30.64^{\mathrm{a}}$ & $19.82^{f}$ \\
\hline Naringenin chalcone & $1.32^{\mathrm{a}}$ & nd & nd & nd & nd & nd \\
\hline Quercetin & $23.46^{\mathrm{e}}$ & $24.28^{d}$ & $29.64^{c}$ & $32.59^{b}$ & $37.13^{\mathrm{a}}$ & $24.03^{f}$ \\
\hline Chlorogenic acid & nd & $42.25^{c}$ & $42.25^{c}$ & $46.88^{b}$ & $59.78^{a}$ & $42.58^{d}$ \\
\hline Myricetin & $21.23^{f}$ & $28.41^{e}$ & $32.08^{c}$ & $32.55^{b}$ & $38.41^{a}$ & $30.64^{d}$ \\
\hline Isorhamnetin & $2.10^{\mathrm{a}}$ & $0.03^{c}$ & $0.05^{\mathrm{b}}$ & $0.03^{b}$ & $0.02^{\mathrm{b}}$ & $0.02^{b}$ \\
\hline 3,5-dicaffeoylquinic acid & $1.10^{f}$ & $27.17^{e}$ & $29.64^{c}$ & $35.30^{b}$ & $41.59^{a}$ & $28.89^{d}$ \\
\hline 4,5-dicaffeoylquinic acid & $2.65^{e}$ & $39.96^{c}$ & $42.25^{c}$ & $44.20^{\mathrm{b}}$ & $51.28^{\mathrm{a}}$ & $37.32^{d}$ \\
\hline Rutin & $6.51^{\mathrm{a}}$ & nd & nd & nd & nd & nd \\
\hline Total & $211.18^{f}$ & $233.55^{d}$ & $257.73^{c}$ & $285.33^{\mathrm{b}}$ & $331.81^{\mathrm{a}}$ & $230.86^{\mathrm{e}}$ \\
\hline
\end{tabular}

Key: 100\% Plantain flour; PSC: Plantain: Soycake (70:30\%); PSR: Plantain: Soycake: Rice bran (65:30:5\%); PSO: Plantain: Soycake: Oat bran (65:30:5\%); PSRO: Plantain: Soycake: Rice bran: Oat bran (60:30:5:5); Cerolina: a commercial flour meal; Nd: Below detectable limit

radicals' formation and oxidation of molecules against DPPH. ABTS, $\mathrm{Fe}^{2+}$ chelation, FRAP and $\mathrm{OH}$ free radical increased. However, the PSRO sample had the highest antioxidant activity when compared to other formulated food samples including $100 \%$ plantain flour, but comparable to that of Cerolina (a control sample). The antioxidative activity $\left(\mathrm{IC}_{50}\right)$ of the formulated food sample extracts varied as follows: ABTS $(52.67-61.23 \mathrm{mg} /$ $\mathrm{mL})$, DPPH $(35.45-65.29 \mathrm{mg} / \mathrm{mL})$, FRAP $(57.71-60.04$ $\mathrm{mg} / \mathrm{mL}), \mathrm{Fe}^{2+}$ chelation $(47.29-71.05 \mathrm{mg} / \mathrm{mL})$ and $\mathrm{OH}$

Table 6 Functional and pasting properties of plantain, soycake, rice bran and oat bran composite flour and control samples

\begin{tabular}{|c|c|c|c|c|c|c|}
\hline Samples & $100 \%$ Plantain & PSC & PSR & PSO & PSRO & Cerolina \\
\hline $\mathrm{BD}(\mathrm{g} / \mathrm{mL})$ & $0.74 \pm 0.03^{\mathrm{a}}$ & $0.67 \pm 0.00^{b}$ & $0.71 \pm 0.03^{\mathrm{ab}}$ & $0.71 \pm 0.01^{\mathrm{ab}}$ & $0.67 \pm 0.03^{b}$ & $0.73 \pm 0.01^{a}$ \\
\hline WAC $(\mathrm{g} / \mathrm{mL})$ & $2.50 \pm 0.03^{b}$ & $2.45 \pm 0.05^{b}$ & $2.08 \pm 0.03^{d}$ & $2.15 \pm 0.03^{c}$ & $2.70 \pm 0.05^{a}$ & $2.48 \pm 0.02^{b}$ \\
\hline SC (\%) & $51.43 \pm 0.01^{c}$ & $55.00 \pm 0.01^{a}$ & $48.61 \pm 0.02^{\mathrm{e}}$ & $50.00 \pm 0.01^{d}$ & $53.75 \pm 0.02^{b}$ & $40.63 \pm 0.01^{f}$ \\
\hline LGC (\%) & $8.00 \pm 0.00^{a}$ & $8.00 \pm 0.00^{a}$ & $6.00 \pm 0.00^{c}$ & $6.00 \pm 0.00^{c}$ & $7.00 \pm 0.00^{b}$ & $8.00 \pm 0.00^{a}$ \\
\hline \multicolumn{7}{|l|}{ Pasting Properties } \\
\hline Peak (RVU) & $2186 \pm 0.06^{\mathrm{a}}$ & $584 \pm 0.04^{c}$ & $422 \pm 0.02^{f}$ & $459 \pm 0.05^{e}$ & $500 \pm 0.05^{d}$ & $792 \pm 0.06^{\mathrm{b}}$ \\
\hline Trough (RVU) & $2065 \pm 0.05^{a}$ & $500 \pm 0.03^{c}$ & $362 \pm 0.04^{f}$ & $395 \pm 0.03^{e}$ & $432 \pm 0.06^{d}$ & $571 \pm 0.05^{\mathrm{b}}$ \\
\hline Breakdown (RVU) & $121 \pm 0.03^{b}$ & $84 \pm 0.02^{c}$ & $60 \pm 0.02^{f}$ & $64 \pm 0.04^{\mathrm{e}}$ & $68 \pm 0.07^{d}$ & $221 \pm 0.03^{\mathrm{a}}$ \\
\hline Final Viscosity (RVU) & $3798 \pm 0.16^{a}$ & $996 \pm 0.11^{\mathrm{b}}$ & $717 \pm 0.13^{f}$ & $848 \pm 0.12^{\mathrm{e}}$ & $879 \pm 0.15^{d}$ & $988 \pm 0.16^{c}$ \\
\hline Set back (RVU) & $1733 \pm 0.03^{\mathrm{a}}$ & $496 \pm 0.03^{b}$ & $355 \pm 0.07^{f}$ & $453 \pm 0.02^{c}$ & $447 \pm 0.08^{d}$ & $417 \pm 0.05^{\mathrm{e}}$ \\
\hline Peak Time (Min) & $7 \pm 0.02^{\mathrm{a}}$ & $7 \pm 0.02^{a}$ & $7 \pm 0.04^{a}$ & $7 \pm 0.03^{a}$ & $7 \pm 0.02^{a}$ & $5.27 \pm 0.02^{b}$ \\
\hline Pasting Temp. $\left({ }^{\circ} \mathrm{C}\right)$ & $87.3 \pm 0.03^{d}$ & $92 \pm 0.06^{c}$ & $94.45 \pm 0.06^{\mathrm{a}}$ & $93.65 \pm 0.06^{b}$ & $94.40 \pm 0.06^{\mathrm{a}}$ & $86.45 \pm 0.02^{e}$ \\
\hline
\end{tabular}

Means ( \pm SEM) with different alphabetical superscripts in the same column are significantly different at $P<0.05$

Key: PLT: 100\% Plantain; PSC: Plantain: Soycake (70:30\%); PSR: Plantain: Soycake: Rice bran (65:30:5\%); PSO: Plantain: Soycake: Oat bran (65:30:5\%); PSRO: Plantain: Soycake: Rice bran: Oat bran (60:30:5:5); CTRL: 100\% Commercial dough meal. I*: lightness/darkness; $a^{*}$ : red/green; $b^{*}$ : yellow/blue; $c^{*}$ : brightness/dullness; $h^{*}$ : hue; $\Delta \mathrm{E}$ : total difference; WI: Degree of whiteness 
Table 7 Antioxidant activities of plantain, soycake, rice-bran and oat-bran flour and control samples

\begin{tabular}{|c|c|c|c|c|c|c|}
\hline \multicolumn{5}{|l|}{ Concentration } & \multirow[b]{2}{*}{ DAAI } & \multirow[b]{2}{*}{ R-square } \\
\hline Sample & $50.00 \mathrm{mg} / \mathrm{mL}$ & $75.00 \mathrm{mg} / \mathrm{mL}$ & $100.00 \mathrm{mg} / \mathrm{mL}$ & $\mathrm{IC}_{50} \mathrm{mg} / \mathrm{mL}$ & & \\
\hline \multicolumn{7}{|c|}{ ABTS Free Radical Scavenging Activities (mg TEAC/g) } \\
\hline 100\% Plantain & $0.0031 \pm 0.0001^{9}$ & $0.0078 \pm 0.0003^{9}$ & $0.0125 \pm 0.0001^{e}$ & $64.35^{\mathrm{a}}$ & 1.55 & 0.9507 \\
\hline PSC & $0.0040 \pm 0.0001^{f}$ & $0.0085 \pm 0.0000^{f}$ & $0.0130 \pm 0.0012^{d}$ & $61.23^{\mathrm{b}}$ & 1.63 & 0.9429 \\
\hline PSR & $0.0058 \pm 0.0000^{d}$ & $0.0100 \pm 0.0001^{d}$ & $0.0141 \pm 0.0013^{c}$ & $55.66^{\mathrm{d}}$ & 1.80 & 0.9349 \\
\hline PSO & $0.0060 \pm 0.0001^{c}$ & $0.0101 \pm 0.0001^{c}$ & $0.0142 \pm 0.0001^{c}$ & $58.12^{d}$ & 1.72 & 0.9313 \\
\hline PSRO & $0.0069 \pm 0.0001^{b}$ & $0.0109 \pm 0.0002^{b}$ & $0.0148 \pm 0.0003^{b}$ & $52.67^{e}$ & 1.90 & 0.9306 \\
\hline Cerolina & $0.0049 \pm 0.0000^{\mathrm{e}}$ & $0.0096 \pm 0.0001^{\mathrm{e}}$ & $0.0140 \pm 0.0010^{c}$ & $58.76^{c}$ & 1.70 & 0.9447 \\
\hline Trolox & $0.0091 \pm 0.0000^{\mathrm{a}}$ & $0.0125 \pm 0.0011^{a}$ & $0.0162 \pm 0.0002^{\mathrm{a}}$ & $47.26^{f}$ & 2.12 & 0.9104 \\
\hline \multicolumn{7}{|c|}{ DPPH Free Radical Scavenging Activities (\%) } \\
\hline 100\% Plantain & $2.92 \pm 0.05^{9}$ & $12.08 \pm 0.04^{9}$ & $22.67 \pm 0.05^{9}$ & $72.26^{\mathrm{a}}$ & 1.38 & 0.9669 \\
\hline PSC & $10.69 \pm 0.05^{f}$ & $28.57 \pm 0.04^{f}$ & $46.46 \pm 0.05^{f}$ & $65.29^{\mathrm{b}}$ & 1.53 & 0.9533 \\
\hline PSR & $17.59 \pm 0.04^{d}$ & $37.61 \pm 0.05^{d}$ & $55.43 \pm 0.04^{d}$ & $60.22^{d}$ & 1.66 & 0.9524 \\
\hline PSO & $26.51 \pm 0.04^{c}$ & $41.19 \pm 0.05^{c}$ & $55.87 \pm 0.04^{c}$ & $52.26^{e}$ & 1.91 & 0.9271 \\
\hline PSRO & $53.77 \pm 0.05^{b}$ & $62.95 \pm 0.04^{b}$ & $72.24 \pm 0.05^{b}$ & $35.45^{f}$ & 2.82 & 0.9094 \\
\hline Cerolina & $13.77 \pm 0.04^{e}$ & $31.02 \pm 0.05^{e}$ & $48.27 \pm 0.05^{e}$ & $62.40^{c}$ & 1.60 & 0.9457 \\
\hline Glutathione & $77.85 \pm 0.05^{\mathrm{a}}$ & $80.28 \pm 0.05^{\mathrm{a}}$ & $83.13 \pm 0.04^{\mathrm{a}}$ & $17.51^{9}$ & 5.71 & 0.8798 \\
\hline \multicolumn{7}{|c|}{ FRAP Inhibitory Activities (mg AAE/ g) } \\
\hline $100 \%$ Plantain & $14.24 \pm 0.03^{9}$ & $25.14 \pm 0.03^{9}$ & $42.80 \pm 0.02^{9}$ & $60.93^{\mathrm{a}}$ & 1.64 & 0.8832 \\
\hline PSC & $15.42 \pm 0.03^{f}$ & $30.06 \pm 0.02^{f}$ & $47.21 \pm 0.02^{f}$ & $60.04^{c}$ & 1.67 & 0.9241 \\
\hline PSR & $16.74 \pm 0.02^{\mathrm{d}}$ & $33.65 \pm 0.03^{d}$ & $50.02 \pm 0.03^{d}$ & $59.68^{d}$ & 1.68 & 0.9430 \\
\hline PSO & $17.32 \pm 0.02^{c}$ & $34.71 \pm 0.02^{c}$ & $50.35 \pm 0.03^{c}$ & $58.91^{e}$ & 1.70 & 0.9490 \\
\hline PSRO & $18.04 \pm 0.02^{b}$ & $36.70 \pm 0.02^{b}$ & $50.75 \pm 0.02^{b}$ & $57.71^{f}$ & 1.73 & 0.9626 \\
\hline Cerolina & $15.86 \pm 0.02^{e}$ & $30.48 \pm 0.03^{e}$ & $47.57 \pm 0.03^{e}$ & $60.54^{\mathrm{b}}$ & 1.65 & 0.9234 \\
\hline Ascorbic acid & $18.88 \pm 0.04^{\mathrm{a}}$ & $37.56 \pm 0.05^{\mathrm{a}}$ & $51.59 \pm 0.05^{\mathrm{a}}$ & $57.20^{9}$ & 1.75 & 0.9621 \\
\hline \multicolumn{7}{|c|}{$\mathrm{Fe}^{2+}$ Chelation Activities $(\mathrm{mg} / \mathrm{mL}$ ) } \\
\hline $100 \%$ Plantain & $4.25 \pm 0.10^{9}$ & $17.19 \pm 0.10^{f}$ & $37.90 \pm 0.10^{f}$ & $75.95^{\mathrm{a}}$ & 1.32 & 0.9691 \\
\hline PSC & $8.44 \pm 0.09^{f}$ & $17.19 \pm 0.11^{f}$ & $37.07 \pm 0.10^{f}$ & $71.05^{\mathrm{b}}$ & 1.41 & 0.8727 \\
\hline PSR & $15.98 \pm 0.11^{d}$ & $27.96 \pm 0.10^{d}$ & $39.82 \pm 0.08^{d}$ & $56.24^{d}$ & 1.78 & 0.9343 \\
\hline PSO & $24.49 \pm 0.10^{c}$ & $31.43 \pm 0.10^{c}$ & $43.41 \pm 0.07^{c}$ & $50.51^{e}$ & 1.98 & 0.8428 \\
\hline PSRO & $26.17 \pm 0.07^{b}$ & $39.46 \pm 0.03^{b}$ & $51.91 \pm 0.10^{b}$ & $47.29^{f}$ & 2.11 & 0.9323 \\
\hline Cerolina & $12.87 \pm 0.10^{e}$ & $26.05 \pm 0.07^{e}$ & $38.14 \pm 0.10^{e}$ & $59.33^{c}$ & 1.69 & 0.9478 \\
\hline Ascorbic acid & $39.25 \pm 0.08^{a}$ & $50.64 \pm 0.09^{\mathrm{a}}$ & $60.27 \pm 0.12^{\mathrm{a}}$ & $42.00^{9}$ & 2.38 & 0.9354 \\
\hline \multicolumn{7}{|c|}{ OH Free Radical Scavenging Activities (\%) } \\
\hline 100\% Plantain & $0.77 \pm 0.19^{9}$ & $16.26 \pm 0.13^{f}$ & $52.75 \pm 0.13^{9}$ & $77.24^{a}$ & 1.29 & 0.9905 \\
\hline PSC & $3.63 \pm 0.06^{f}$ & $16.26 \pm 0.13^{f}$ & $54.28 \pm 0.12^{f}$ & $76.65^{b}$ & 1.30 & 0.9996 \\
\hline PSR & $14.07 \pm 0.12^{d}$ & $36.04 \pm 0.12^{d}$ & $57.80 \pm 0.12^{d}$ & $64.53^{d}$ & 1.55 & 0.9522 \\
\hline PSO & $29.67 \pm 0.13^{c}$ & $42.42 \pm 0.12^{c}$ & $64.39 \pm 0.13^{c}$ & $55.66^{\mathrm{e}}$ & 1.80 & 0.9393 \\
\hline PSRO & $32.75 \pm 0.12^{b}$ & $57.14 \pm 0.13^{b}$ & $80.00 \pm 0.13^{b}$ & $54.20^{f}$ & 1.85 & 0.8548 \\
\hline Cerolina & $8.35 \pm 0.13^{e}$ & $32.53 \pm 0.12^{e}$ & $54.73 \pm 0.12^{e}$ & $68.79^{c}$ & 1.45 & 0.9722 \\
\hline Mannitol & $46.59 \pm 0.08^{a}$ & $66.58 \pm 0.09^{a}$ & $88.63 \pm 0.15^{a}$ & $49.43^{9}$ & 2.02 & 0.9113 \\
\hline
\end{tabular}

Means ( $($ SEM) with different alphabetical superscripts in the same column are significantly different at $P<0.05$. Key: PSC: Plantain: Soycake (70:30\%); PSR: Plantain: Soycake: Rice bran (65:30:5\%); PSO: Plantain: Soycake: Oat bran (65:30:5\%); PSRO: Plantain: Soycake: Rice bran: Oat bran (60:30:5:5); Cerolina: Commercial flour meal. DAAl: Dietary antioxidant activity index 
free radicals $(54.79-76.65 \mathrm{mg} / \mathrm{mL})$ for PSRO and PSC, respectively.

\section{Antihyperlipidemic properties}

The blood lipid profile of cholesterol-induced Albino Wistar rats fed on experimental food samples and control sample is presented in Table 8. The plasma lipid profile, that is, total cholesterol TC), triglyceride (TG), high-density lipoprotein concentration (HDL-conc.), low-density lipoprotein concentration (LDL-conc.) and very low-density lipoprotein concentration (VLDL-conc.) of rats fed on the formulated food samples varied as follows: $20.11-34.61 \mathrm{mg} / \mathrm{dL}, \quad 17.45-25.93 \mathrm{mg} / \mathrm{dL}, \quad 12.89-$ 23.72, $\mathrm{mg} / \mathrm{dL} 3.73-5.87 \mathrm{mg} / \mathrm{dL}$ and $7.93-11.79 \mathrm{mg} / \mathrm{dL}$, respectively.

\section{Discussion}

The moisture content of the flour samples was lower than recommended value $(<10)$ for flour samples, and this indicates that the flour samples could be stored for longer period. The amount of moisture content in the flour sample determines the growth and activities of microorganisms, hence, storage stability of the flour. Scientific findings have established that high moisture content of flour samples above $10 \%$ facilitate growth of microorganisms and food spoilage, whereas, low moisture content inhibits food spoilage and retain nutritional quality of the food products $[71,72]$. The protein and fiber content of the present study food products were significantly $(<0.05)$ higher than $100 \%$ plantain flour, but were comparable to that of Cerolina (a commercial food product) and other similar studies [73, 74]. The high protein and fiber content of the food products could be attributed to the inclusion of soycake, rice-bran and oatbran, which are high in protein and fiber content than $100 \%$ plantain flour. Nutritional studies have established that a regular intake of dietary fibers is beneficial, particularly in preventing arteriosclerosis and chronic diseases like diabetes and hypertension [75, 76]. The protein content of the developed food products was higher than minimum recommended daily requirements ( $>16 \mathrm{~g} /$ day), and this implies that the food products could be suitable for the prevention of protein-energy malnutrition particularly in children.

Mineral content of the food products in this study was comparatively lower than daily-recommended values. This implies that the food products was low in mineral composition; hence, there is a need to complement the food products intake with mineral supplements in order to prevent micronutrient deficiency. This observation was in line with previous studies, which reported that most of the African diets are low in micronutrients [77], which is responsible for high prevalence of micronutrient deficiency in many parts of developing countries including Nigeria. Deficiency of essential minerals like iron, calcium, zinc, phosphorous, sodium and potassium may lead to several health challenges such as bone deformation, anemia, electrolyte imbalance, brain and growth retardation [77].

The EAAI and P-BV of the PSRO sample were higher than other food samples including cerolina and recommended values $(>70 \%)$ for ideal food products. This

Table 8 Effect of dough meal flour from plantain, soycake, rice bran and oat bran dough meal and control sample on blood lipid profile of cholesterol-induced Albino Wistar Rats

\begin{tabular}{|c|c|c|c|c|c|c|c|c|}
\hline Parameters & SAT & $\begin{array}{l}100 \% \\
\text { Plantain }\end{array}$ & PSC & PSR & PSO & PSRO & Cerolina & *NR \\
\hline$\overline{\mathrm{TC}(\mathrm{mg} / \mathrm{dL})}$ & $154.17 \pm 0.38^{a}$ & $26.85 \pm 0.09^{d}$ & $20.11 \pm 0.02^{g}$ & $22.47 \pm 0.08^{f}$ & $28.04 \pm 0.02^{c}$ & $34.78 \pm 0.08^{b}$ & $25.61 \pm 0.02^{e}$ & $<200$ \\
\hline $\mathrm{TG}(\mathrm{mg} / \mathrm{dL})$ & $212.10 \pm 0.14^{a}$ & $18.93 \pm 0.06^{e}$ & $17.45 \pm 0.05^{\mathrm{g}}$ & $18.77 \pm 0.02^{f}$ & $20.53 \pm 0.08^{d}$ & $25.93 \pm 0.04^{c}$ & $28.42 \pm 0.08^{b}$ & $<150$ \\
\hline $\mathrm{HDL}-\mathrm{C}(\mathrm{mg} / \mathrm{dL})$ & $42.64 \pm 0.50^{a}$ & $12.20 \pm 0.06^{\mathrm{g}}$ & $12.89 \pm 0.06^{f}$ & $12.93 \pm 0.01^{e}$ & $17.72 \pm 0.04^{d}$ & $23.72 \pm 0.04^{b}$ & $19.48 \pm 0.02^{c}$ & $<50$ \\
\hline $\mathrm{LDL}-\mathrm{C}(\mathrm{mg} / \mathrm{dL})$ & $64.39 \pm 0.14^{a}$ & $10.86 \pm 0.05^{b}$ & $3.73 \pm 0.04^{f}$ & $5.78 \pm 0.06^{e}$ & $6.21 \pm 0.09^{c}$ & $5.87 \pm 0.05^{d}$ & $0.45 \pm 0.02^{g}$ & $<15$ \\
\hline $\begin{array}{l}\text { VLDL-conc. (mg/ } \\
\mathrm{dL} \text { ) }\end{array}$ & $96.41 \pm 3.10^{a}$ & $8.60 \pm 0.13^{e}$ & $7.93 \pm 0.11^{\mathrm{g}}$ & $8.53 \pm 0.09^{f}$ & $9.33 \pm 0.21^{d}$ & $11.79 \pm 0.10^{c}$ & $12.92 \pm 0.22^{b}$ & \\
\hline \multicolumn{9}{|c|}{ Lipid Health indices of plasma } \\
\hline $\begin{array}{l}\text { Coronary Risk } \\
\text { Index }\end{array}$ & $3.62 \pm 0.0^{a}$ & $2.20 \pm 0.01^{b}$ & $1.56 \pm 0.01^{d}$ & $1.74 \pm 0.00^{c}$ & $1.58 \pm 0.02^{d}$ & $1.47 \pm 0.00^{e}$ & $1.31 \pm 0.01^{f}$ & $\begin{array}{l}3.421- \\
8.454\end{array}$ \\
\hline $\begin{array}{l}\text { Atherogenic } \\
\text { index }\end{array}$ & $1.51 \pm 0.11^{\mathrm{a}}$ & $0.89 \pm 0.00^{b}$ & $0.29 \pm 0.00^{f}$ & $0.45 \pm 0.00^{c}$ & $0.35 \pm 0.01^{d}$ & $0.25 \pm 0.00^{\mathrm{e}}$ & $0.02 \pm 0.00^{9}$ & $\begin{array}{l}2.622- \\
6.623\end{array}$ \\
\hline $\log (\mathrm{TG} / \mathrm{HDL}-\mathrm{C})$ & $0.69 \pm 0.00^{a}$ & $0.19 \pm 0.00^{b}$ & $0.13 \pm 0.00^{e}$ & $0.16 \pm 0.00^{d}$ & $0.06 \pm 0.00^{f}$ & $0.04 \pm 0.00^{g}$ & $0.16 \pm 0.01^{c}$ & $0.11-0.21$ \\
\hline \multicolumn{9}{|c|}{ Food intake and changes in body weight of experimental animals ( $g$ ) } \\
\hline Food intake & $1291.02 \pm 5.00^{c}$ & $472.00 \pm 7.10^{f}$ & $1142.00 \pm 4.10^{\mathrm{e}}$ & $1403.00 \pm 5.20^{a}$ & $1289.00 \pm 4.50^{c}$ & $1239.00 \pm 3.32^{d}$ & $1344.00 \pm 3.20^{b}$ & - \\
\hline Weight gain & $37.14 \pm 2.31^{b}$ & $-8.70 \pm 0.03^{f}$ & $25.70 \pm 2.01^{d}$ & $24.20 \pm 3.03^{e}$ & $37.10 \pm 2.11^{b}$ & $29.20 \pm 3.01^{c}$ & $41.50 \pm 2.01^{a}$ & - \\
\hline
\end{tabular}

Key: SAT: rats fed with basal diet + Satin; PLT: rats fed with $\% 100$ Plantain; PSC rats fed with (Plantain $70 \%+$ Soycake $30 \%$ ); PSR: rats fed with (Plantain $65 \%+$ Soycake 30\% + Rice bran 5\%); PSO: rats fed with (Plantain 65\% + Soycake 30\% + Oat bran 5\%); PSRO: rats fed wit (Plantain 60\% + Soycake 30\%+ Oat bran 5\% + Rice bran 5\%) and Cerolina. *NR: American Diabetes Association [124] 
indicates that PSRO blend could be suitable as a food product to support growth in children and physiological maintenance in adults. Studies have reported that regular intakes of branched chain amino acids are health beneficial, particularly in the management of type - 2 diabetes [78]. Also, it evident that arginine intakes plays an important role in the production of nitric oxide in the body, which helps to relax arteries for the free flow of blood, hence reduces the risk of high blood pressure [79]. These findings further established nutritional quality and health benefits of the present study food products in managing diabetes and hypertension.

The phytochemical concentrations in food products in this present study were low; and this implies that the experimental food products could be suitable for human consumption without any side effects or interfering with other vital nutrients. In recent times, studies have shown that regular consumption of bioactive phytochemicals such as phenol, flavonoid, tannin, etc. in functional foods at low concentration proffers health benefits [80]. For instance, it is evident that regular consumption of plantbased foods containing flavonoid and phenolic compounds prevent degenerative chronic diseases like hypertension and diabetes; and these health benefits are attributed to antioxidant properties of these phytochemicals [80]. Antioxidants are chemical compounds capable of inhibiting the oxidation of cell molecules [81], and thereby preventing oxidative stress in animals.

Studies have shown that these phenolic compounds have antioxidative properties and ability to scavenge free radicals; hence, ability to prevent the occurrence of oxidative stress and associated chronic degenerative diseases like diabetes, etc. [82, 83]. In recent years, there is a shift of interest from using synthetic antioxidants towards natural antioxidants, due to their non-side effects [84-86]. In view of this, it may be deduced in this study that the formulated food samples are suitable as exogenous antioxidants diets, and that their regular intakes may enhance endogenous antioxidants activities to fight against excessive production of free radicals in the body [87].

The variation in BD between the blended flour samples and that of $100 \%$ plantain flour and Cerolina could be attributed to the inclusion of rice-bran and oat-bran inclusion, which mighty have influenced the properties of starch. Bulk density indicates the porosity of a product, and it determining the type of packaging material required [51]. It is well established that the higher the bulk density of flour sample, the denser the packaging material required [51]. The variation between WAC of these flour samples could be attributed to differences in the protein content, degree of interaction with water and conformational characteristics [88]. The low values of $\mathrm{BD}$ and WAC of the food products in this study have nutritional advantage in maintaining high nutrientenergy density that is of nutritional benefits to the young children with small stomach size $[89,90]$. However, flour sample with high water absorption may have more hydrophilic constituents like polysaccharides and protein, and more important in baking applications [91].

The variation in the gelling properties of these flour samples could be ascribed to different ratio of proteins, carbohydrates and lipids in their components. The lower the LGC, the better the gelating ability of the protein ingredient [92] and the swelling ability of the flour [93]. The LGC of the flour samples in this study was similar to the report of Chandra et al. [94]. The values of swelling capacity of the flour samples varied from $48.61-55 \%$, and were higher than cerolina, but within the same range value to that of $100 \%$ plantain flour. The variation in the swelling capacity of the flours in this study could be attributed to particle sizes, processing methods or unit operations.

Pasting property (an index for predicting the ability of a food to form a paste when subjected to heat applications) is one of the most important properties that influence quality and aesthetic properties of food products $[95,96]$. The low peak viscosity value that was observed in the experimental flour samples could be attributed to the inclusion of soycake, rice-bran and oat-bran flour into plantain-based composite flour samples. High peak viscosity of processing flour reflects fragility of the swollen granules, which first swell and then breaks down under the continuous mixing of the Rapid Visco Analyzer. The low peak viscosity values noted in this study is of processing advantage, particularly for the preparation of less stiff dough products [97].

The trough viscosity values observed in this present study indicate the tendency of the plantain-based flour samples to breakdown during cooking. The breakdown viscosity is an index of the stability of the starch and a measure of the ease with which the swollen granules can be disintegrated [98]; and that the higher the breakdown viscosity, the lower the ability of the flour to withstand heating and shear stress during cooking [99].

These parameters, i.e., final viscosity, setback, peak time and pasting temperature are very important factors in determining the quality of food products. For instance, final viscosity indicates the ability of starchy foods to form viscous paste after cooking and cooling, and it is an important quality parameter used in predicting the final textural quality of starchy foods [100]. High setback values affect dough digestibility [101], while lower value indicates lower tendency of starch retrogradation [102]. The pasting temperature measures the minimum temperature needed to cook a given food sample [103], while that of peak time measures the cooking time [99] of starch. 
An $\mathrm{IC}_{50}$ value is the measure of the effectiveness of a substance in a biochemical reaction to result in $50 \%$ antioxidant activities, the smaller the $\mathrm{IC}_{50}$ value the higher the antioxidant activity [104]. The low $\mathrm{IC}_{50}$ value observed in PSRO further established the potency of the food product as an exogenous antioxidant diet. The antioxidative ability of this food sample (PSRO) could be attributed to the synergetic effect of the flour sample combinations i.e., plantain, Soycake, oat-bran and rice-bran. Studies have earlier reported that oat-bran [105], rice-bran [106] and soycake [107], which are the most active ingredients in these formulation contain appreciable amount of bioactive compounds with antioxidant activities [108, 109].

Comparatively, the concentration of plasma lipid profile of rats fed on the formulated diets was similar to those rats fed on $100 \%$ plantain flour and Cerolina, but lower than those rats in group SAT (rats treated with Satin, an anticholesterol agent). For the health lipid indices, the values of $\log$ (TG/HDL-conc.), atherogenic index (AI) and coronary risk index (CRI) ranged from $0.25-0.89$ and $1.47-2.20$, respectively; and were lower than normal recommended values for AI (4.4-7.1) and CRI (3.4-4.0) [110]. This finding agreed with the report of Hasanein et al. [111], who reported that the AI (2.622-6.623) and CRI (3.421-8.454) of obese rats treated with tea and cinnamon extract. The decreased in total cholesterol and low-density lipoprotein (LDL) levels couple with increased in high-density lipoprotein (HDL) levels that were observed in obese rats fed on the experimental diets in this present study could be considered health benefits for the consumers. Recently, epidemiological studies have implicated high level of total cholesterol, LDL-cholesterol, or low HDL-cholesterol as the major risk factors for the atherosclerosis-related diseases (cardiovascular diseases, diabetes, etc.) [112-114]; and are major targets for the treatment and prevention of atherosclerosis diseases [115-117].

\section{Conclusion}

This study developed and evaluated, nutritional, bioactive phytochemicals, antioxidants activities, and antihyperlipidemic properties of food from plantain, soycake, oat-bran and rice-bran flour. The finding established consumption of high fats diets - rich in low density lipoproteins should be avoided. As they expose the body to risk of arteriosclerosis formation. However, formulated food samples, particularly PSRO (Plantain 60\%, Soycake $30 \%$, Rice bran $5 \%$, Oat bran $5 \%$ ), had higher antioxidant and anti-hyperlipidemia activities when compared with $100 \%$ plantain flour and Cerolina (a commercial flour). Hence, this food product, that is, PSRO, may be suitable as functional food for the treatment and prevention of arteriosclerosis formation, hence, hypertension.

\section{Acknowledgments}

The authors wish to acknowledge and appreciate the Tertiary Education Trust Fund (TETFUND) of the Federal Government of Nigeria. Richardson Milling Limited, Portage La Prairie, Manitoba (MB) for provision of oat bran.

\section{Authors' contributions}

IJAROTIMI Oluwole Steve \& FAGBEMI Tayo Nathaniel designed the research; while implementation of the research, data analyses and manuscript preparation were done by OLUWAJUYITAN Timilehin David and IJAROTIMI Oluwole Steve. All authors read and approved the final manuscript.

\section{Funding}

This research was funded by TETFUND research grant won by Professor T. N. Fagbemi of Food Science and Technology Department, Federal University of Technology Akure, Nigeria (Research ID: VCPU/TETFUND/155C).

\section{Availability of data and materials}

The datasets used and/or analyzed during the current study are available from the corresponding author on reasonable request.

\section{Declarations}

\section{Competing interests}

The authors declared that there were no conflicts of interest for the study.

\section{Ethics approval}

The Ethical Committee for Laboratory Animals of School of Agriculture and Agricultural Technology, Federal University of Technology Akure, Nigeria (Approval number: FUTA/ SAAT/2018/021) approved the study protocol.

\section{Consent for publication}

Not Applicable.

Received: 18 January 2021 Accepted: 15 November 2021

Published online: 26 November 2021

References

1. Tony L, Areekal B, Surendran NAT, Ramachandran R, Philip RR, Rajasi RS. Prevalence of hypertension and pre-hypertension among adolescent schoo children in Thiruvananthapuram, Kerala, India. Int J Community Med Public Health. 2016. https://doi.org/10.18203/2394-6040.ijcmph20164291.

2. Jia X, Lorenz P, Ballantyne CM. Poststatin Lipid Therapeutics: A Review. Methodist Debakey Cardiovasc J. 2019. https://doi.org/10.14797/mdcj-1 $5-1-3$

3. Aounallah-Skhiri H, El Ati J, Traissac P, Romdhane HB, Eymard-Duvernay S, Delpeuch F, Achour N, Maire B. Blood pressure and associated factors in a North African adolescent population. a national cross-sectional study in Tunisia. BMC Public Health. 2012; https://doi.org/10.1186/1471-2458-12-98, Blood pressure and associated factors in a North African adolescent population. a national cross-sectional study in Tunisia.

4. Ajayi 1O, Soyannwo MAO, Asinobi AO, Afolabi NB, Ayede Al, Bamgboye EA. Blood pressure pattern and hypertension related risk factors in an urban community in Southwest Nigeria: the Mokola hypertension initiative project, Ibadan. Nigeria J Public Health Epidemiol. 2017;9(4):51-64. https://doi.org/1 0.5897/JPHE2017.0908

5. van de Vijver S, Akinyi H, Oti S, Olajide A, Agyemang C, Aboderin I, et al. Status report on hypertension in Africa-consultative review for the 6th session of the African union conference of ministers of health on NCD's. PAMJ. 2014;16:1-17

6. Iwelunmor J, Airhihenbuwa CO, Cooper R, Tayo B, Plange-Rhule J, Adanu R, et al. Prevalence, determinants and systems-thinking approaches to optimal hypertension control in West Africa. Glob Health. 2014;10(1):42. https://doi. org/10.1186/1744-8603-10-42.

7. Noubiap JJN, Bigna JJR, Nansseu JRN. Low sodium and high potassium intake for cardiovascular prevention: evidence revisited with emphasis on challenges in sub-Saharan Africa. J Clin Hypertens. 2015;17(1):81-3. https:// doi.org/10.1111/jch.12439.

8. Jaja T, Oduwole AO, Fetuga B, Abdus-Salam IA. Prevalence of prediabetes in secondary school students in Port Harcourt. Nigeria Afr J Diabetes Med. 2015;23:1-4. 
9. Odey FA, Ekanem EE, Udoh AE, Ntui I. Lipid profiles of apparently healthy adolescents in Calabar. Nigeria Central Afr J Med. 2007;53(1-4). https://doi. org/10.4314/cajm.v53i1-4.62605.

10. Popkin BM, Gordon-Larsen P. The nutrition transition: worldwide obesity dynamics and their determinants. Int J Obes. 2004;28(S3):S2-9. https://doi. org/10.1038/sj.ijo.0802804.

11. Benson J, Britten N. What effects do patients feel from their antihypertensive tablets and how do they react to them? Qualitative analysis of interviews with patients. Fam Pract. 2006;23(1):80-7. https://doi. org/10.1093/fampra/cmi081.

12. Soliman GA. Dietary fiber, atherosclerosis, and cardiovascular disease. Nutrients. 2019;11(5). https://doi.org/10.3390/nu11051155.

13. Ferrari CK. Functional foods, herbs and nutraceuticals: towards biochemical mechanisms of healthy aging. Biogerontol. 2004;5(5):275-90. https://doi. org/10.1007/s10522-004-2566-Z.

14. Barta I, Smerak P, Polivkova Z, Sestakova H, Langova M, Turek B, et al. Current trends and perspectives in nutrition and cancer prevention. Neoplasma. 2006. 1016416008.

15. Hasler CM, Brown AC. Position of the American dietetic association: functional foods. J Am Diet Assoc. 2009;109(4):735-46. https://doi.org/10.101 6/j.jada.2009.02.023.

16. Amigo-Benavent M, Silván JM, Moreno FJ, Villamiel M, Del Castillo MD. Protein quality, antigenicity, and antioxidant activity of soy-based foodstuffs. J Agric Food Chem. 2008;56(15):6498-505. https://doi.org/10.1021/jf800697n.

17. Liu Z, Ren Z, Zhang J, Chuang CC, Kandaswamy E, Zhou T, et al. Role of ROS and nutritional antioxidants in human diseases. Front Physiol. 2018;9. https://doi.org/10.3389/fphys.2018.00477.

18. Borbalan AMA, Zorro L, Guillen DA, Barroso CG. Study of the polyphenol content of red and white grape varieties by liquid chromatography-mass spectrometry and its relationship to antioxidant power. J Chromatogr A. 2003;1012(1):31-8. https://doi.org/10.1016/50021-9673(03)01187-7.

19. Fernández-Pachón MS, Villano D, Garcla-Parrilla MC, Troncoso AM. Antioxidant activity of wines and relation with their polyphenolic composition. Anal Chim Acta. 2004;513(1):113-8. https://doi.org/10.1016/j.aca.2004.02.028.

20. Ploetz RC, Kepler AK, Daniells J, Nelson SC. Banana and plantain —an overview with emphasis on Pacific island cultivars. Species Profiles Pacif Island Agrof. 2007:21-32.

21. Tribess TB, Hernández-Uribe JP, Méndez-Montealvo MGC, Menezes EW, Bello-Perez LA, Tadini CC. Thermal properties and resistant starch content of green banana flour (Musa cavendishii) produced at different drying conditions. LWT-Food Sci Technol. 2009;42(5):1022-5. https://doi.org/10.101 6/j.Iwt.2008.12.017

22. Akubor PI, Ishiwu C. Chemical composition, physical and sensory properties of cakes supplemented with plantain peel flour. Int J Agric Policy Res. 2013; 1(Suppl 4):87-92.

23. Ojewole JAO, Adewunmi CO. Hypoglycemic effect of methanolic extract of Musa paradisiaca (Musaceae) green fruits in normal and diabetic mice. Meth Findings Exper Clin Pharmacol. 2003;25(Suppl 6):453-6. https://doi.org/10.13 58/mf.2003.25.6.769651.

24. Vivienne I, Uchenna O, Peace A, Clinton O. Composition and sensory properties of plantain cake. Afr J Food Sci. 2016;10(Suppl 2):25-32. https:// doi.org/10.5897/AJFS2015.1292.

25. Juarez-Garcia E, Agama-Acevedo E, Sáyago-Ayerdi SG, Rodriguez-Ambriz SL, Bello-Perez LA. Composition, digestibility and application in bread making of banana flour. Plant Foods Hum Nutr. 2006;61(3):131-7. https://doi.org/1 0.1007/s11130-006-0020-X.

26. Mastromatteo M, Danza A, Lecce L, Spinelli S, Lampignano V, Laverse J, et al. A Effect of durum wheat varieties on bread quality. Int J Food Sci Technol. 2014:49(1):72-81. https://doi.org/10.1111/ijfs.12276.

27. Singh AMIT, Dubey RACHIYATA, Paliwal RT, Saraogi GK, Singhai AK. Nutraceuticals-an emerging era in the treatment and prevention of diseases. Int J Pharm Pharm Sci. 2012;4(Suppl 4):39-43.

28. Oluwajuyitan TD, ljarotimi OS. Nutritional, antioxidant, glycaemic index and Antihyperglycaemic properties of improved traditional plantain-based (Musa AAB) dough meal enriched with tigernut (Cyperus esculentus) and defatted soybean (Glycine max) flour for diabetic patients. Heliyon. 2019;5(4):e01504. https://doi.org/10.1016/j.heliyon.2019.e01504.

29. Oluwajuyitan TD, ljarotimi OS, Fagbemi TN. Nutritional, biochemical and organoleptic properties of high protein-fibre functional foods developed from plantain, defatted soybean, rice-bran and oat-bran flour. Nutr Food Sci. 2020;51(4):704-24. https://doi.org/10.1108/NFS-06-2020-0225.
30. Qureshi AA, Sami SA, Khan FA. Effects of stabilized rice bran, its soluble and fiber fractions on blood glucose levels and serum lipid parameters in humans with diabetes mellitus types I and II. J Nutr Biochem. 2002;13(3): 175-87. https://doi.org/10.1016/S0955-2863(01)00211-X.

31. Rondanelli M, Opizzi A, Monteferrario F, Klersy C, Cazzola R, Cestaro B. Betaglucan-or rice bran-enriched foods: a comparative crossover clinical trial on lipidic pattern in mildly hypercholesterolemic men. Eur J Clin Nutr. 2011; 65(7):864-71. https://doi.org/10.1038/ejcn.2011.48.

32. Meijer GW, Bressers MA, Arjan de Groot W, Rudrum M.Effect of struture and form on the ability of plant sterols to inhibit cholesterol absorption in hamsters. Lipids. 2003; https://doi.org/10.1007/s11745-003-1119-4, Effect of struture and form on the ability of plant sterols to inhibit cholesterol absorption in hamsters.

33. Wilson TA, Nicolosi RJ, Woolfrey B, Kritchevsky D. Rice bran oil and oryzanol reduce plasma lipid and lipoprotein cholesterol concentrations and aortic cholesterol ester accumulation to a greater extent than ferulic acid in hypercholesterolemic hamsters. J Nutr Biochem. 2007;18(2):105-12. https:// doi.org/10.1016/j.jnutbio.2006.03.006.

34. Glore SR, Van Treeck D, Knehans AW, Guild M. Soluble fiber and serum lipids: a literature review. J Am Diet Assoc. 1994;94(4):425-36. https://doi. org/10.1016/0002-8223(94)90099-X.

35. Contreras F, Rivera M, Vasquez J, De la Parte MA, Velasco M. Diabetes and hypertension physiopathology and therapeutics. J Hum Hypertens. 2000; 14(S1):S26-31. https://doi.org/10.1038/sj.jhh.1000983.

36. McKeown NM, Meigs JB, Liu S, Saltzman E, Wilson PW, Jacques PF. Carbohydrate nutrition, insulin resistance, and the prevalence of the metabolic syndrome in the Framingham offspring cohort. Diabetes Care. 2004;27(2):538-46. https://doi.org/10.2337/diacare.27.2.538.

37. Odom TC, Udensi EA, Ogbuji CA. Evaluation of hypoglycemic properties of Mucuna cochichinensis unripe carica papaya and unripe musa paradisiaca flour blends. Eur J Biol Med Sci Res. 2013;1 (Suppl 1):15-22.

38. Famakin O, Fatoyinbo A, ljarotimi OS, Badejo AA, Fagbemi TN. Assessment of nutritional quality, glycaemic index, antidiabetic and sensory properties of plantain (Musa paradisiaca)-based functional dough meals. J Food Sci Technol. 2016;53(11):3865-75. https://doi.org/10.1007/s13197-016-2357-y.

39. Mepba HD, Eboh L, Nwaojigwa SU. Chemical composition, functional and baking properties of wheat-plantain composite flours. Afr J Food, Agric Nutr Develop. 2007;7(Suppl 1):1-23.

40. AOAC. Association of official analytical chemist Official Methods of Analysis of the Analytical Chemist International. 18th ed. Gathersburg: AOAC International 2012; 2012.

41. Mansouri F, Ben Moumen A, Richard G, Fauconnier ML, Sindic M, Elamrani A, et al. Proximate composition, amino acid profile, carbohydrate and mineral content of seed meals from four Safflower (Carthamus tinctorius L.) varieties grown in north-eastern Morocco. Oilseeds Fats Crops Lipids. 2018; 25(2):A202. https://doi.org/10.1051/ocl/2018001.

42. Hartcamp AD, White JW, Rodríguez AA, Bänzinger M, Hernández G, Bates H. Modified method for rapid tryptophan analysis in maize. CIMMYT Res Bull. 2000;13:3-6.

43. Fagbemi TN, Oshodi AA, Ipinmoroti KO. Processing effects on some antinutritional factors and in vitro multienzyme protein digestibility (IVPD) of three tropical seeds: breadnut (Artocarpus altilis), cashewnut (Anacardium occidentale) and fluted pumpkin (Telfairia occidentalis). Pak J Nutr. 2005.

44. Boham BA, Kocipai-Abyazan R. Flavonoids and condensed tannins from leaves of Hawaiian Vaccinium vaticulatum and V. calycinium. Pacif Sci. 1974; 48(Suppl. 4):458-63.

45. Obadoni BO, Ochuko PO. Phytochemical composition, spoilage and shelf life extension. Studies and comparative efficacy of the crude extracts of some homeostatic plants in Edo and Delta states of Nigeria. Glob J Pure Appl Sci. 2001;8:203-8

46. Adeniyi SA, Orjiekwe CL, Ehiagbonare JE, Arimah BD. Preliminary phytochemical analysis and insecticidal activity of ethanolic extracts of four tropical plants (Vernonia amygdalina, Sida acuta, Ocimum gratissimum and Telfaria occidentalis) against beans weevil (Acanthscelides obtectus). Int J Phys Sci. 2010;5(Suppl 6):753-62.

47. Smith MA, Rottkamp CA, Nunomura A, Raina AK, Perry G. Oxidative stress in Alzheimer's disease. Biochim Biophy Acta Molecul Basis Dis. 2000;1502(1): 139-44. https://doi.org/10.1016/S0925-4439(00)00040-5.

48. Georgé $\mathrm{S}$, Brat $\mathrm{P}$, Alter $\mathrm{P}$, Amiot MJ. Rapid determination of polyphenols and vitamin C in plant-derived products. J Agric Food Chem. 2005;53(Suppl 5):1370-3. https://doi.org/10.1021/jf048396b. 
49. Kelley WT, Coffey DL, Mueller TC. Liquid chromatographic determination of phenolic acids in soil. J AOAC Int. 1994;77(4):805-9. https://doi.org/10.1093/ jaoac/77.4.805

50. Provan GJ, Scobbie L, Chesson A. Determination of phenolic acids in plant cell walls by microwave digestion. J Sci Food Agric. 1994;64(1):63-5. https:// doi.org/10.1002/jsfa.2740640110.

51. Iwe MO, Onadipe OO. Effect of addition of extruded full fat soy flour into sweet potato flour on functional properties of the mixture. J Sustain Agric Environ. 2001;3(Suppl 1):109-17.

52. Akpata MI, Miachi OE. Proximate composition and selected functional properties of Detarium microcarpum. Plant Foods Hum Nutr. 2001;56(4): 297-302. https://doi.org/10.1023/A:1011836332105.

53. AACC. Approved methods of the Association of Cereal Chemist (11th ed., method 61-02.01. Determination of the pasting properties of rice with the rapid visco analyzer). Author: St. Paul, MN; 2001.

54. Re R, Pellegrini N, Proteggente A, Pannala A, Yang M, Rice-Evans C. Antioxidant activity applying an improved ABTS radical cation decolorization assay. Free Rad Biol Med. 1999;26(9-10):1231-7. https://doi. org/10.1016/S0891-5849(98)00315-3

55. Aluko RE, Monu E. Functional and bioactive properties of quinoa seed protein hydrolysates. J Food Sci. 2003;68(4):1254-8. https://doi.org/10.1111/ j.1365-2621.2003.tb09635.X.

56. Xie Z, Huang J, Xu X, Jin Z. Antioxidant activity of peptides isolated from alfalfa leaf protein hydrolysate. Food Chem. 2008;111(2):370-6. https://doi. org/10.1016/j.foodchem.2008.03.078.

57. Zhang LL, Lin YM. Tannins from Canarium album with potent antioxidant activity. J Zhejiang Uni Sci B. 2008;9(5):407-15. https://doi.org/10.1631/jzus. B0820002.

58. Girgih AT, Udenigwe CC, Aluko RE. In vitro antioxidant properties of hemp seed (Cannabis sativa L.) protein hydrolysate fractions. J Am Oil Chem Soc. 2011. https://doi.org/10.1007/s11746-010-1686-7.

59. Scherer R, Godoy HT. Antioxidant activity index (AAI) by the 2, 2-diphenyl-1picrylhydrazyl method. Food Chem. 2009;112(3):654-8. https://doi.org/10.1 016/j.foodchem.2008.06.026.

60. CCAC [Canadian Council on Animal Care]. In: Olfert ED, Cross BM, McWilliam AA, editors. Guide to the Care and Use of Experimental Animals, vol. 1. second ed. Ontario: CCAC; 1993.

61. Kim JY, Nolte LA, Hansen PA, Han DH, Ferguson K, Thompson PA, et al. High-fat diet-induced muscle insulin resistance: relationship to visceral fat mass. Am J Physiol Regulat Integrat Comp Physiol. 2000;279(6):R2057-65. https://doi.org/10.1152/ajpregu.2000.279.6.R2057.

62. Vatandoust N, Rami F, Salehi AR, Khosravi S, Dashti G, Eslami G, et al. Novel high-fat diet formulation and streptozotocin treatment for induction of prediabetes and type 2 diabetes in rats. Adv Biomed Res. 2018;7(1):107. https://doi.org/10.4103/abr.abr_8_17.

63. Siedel J, Hägele EO, Ziegenhorn J, Wahlefeld AW. Reagent for the enzymatic determination of serum total cholesterol with improved lipolytic efficiency. Clin Chem. 1983;29(6):1075-80. https://doi.org/10.1093/clinchem/29.6.1075.

64. Tietz NW. Serum triglyceride determination. Clin Guide Lab Tests. 1990;2: 554-6.

65. Jacobs DR Jr, Mebane IL, Bangdiwala SI, Criqui MH, Tyroler HA. High density lipoprotein cholesterol as a predictor of cardiovascular disease mortality in men and women: the follow-up study of the lipid research clinics prevalence study: lipid research clinics program. Am J Epidemiol. 1990; 131(1):32-47. https://doi.org/10.1093/oxfordjournals.aje.a115483.

66. Albers JJ, Warnick GR, Chenng MC. Quantitation of high density lipoproteins. Lipids. 1978;13(Suppl 12):926-32. https://doi.org/10.1007/BF02 533852.

67. Friedewald WT, Levy RI, Fredrickson DS. Estimation of the concentration of low-density lipoprotein cholesterol in plasma, without use of the preparative ultracentrifuge. Clin Chem. 1972;18(6):499-502. https://doi.org/1 0.1093/clinchem/18.6.499.

68. Satheesh MA, Pari L. Effect of pterostilbene on lipids and lipid profiles in streptozotocin-nicotinamide induced type 2 diabetes mellitus. J Appl Biomed. 2008;6(Suppl 1):31-7. https://doi.org/10.32725/jab.2008.005.

69. Williamson EM, Opako DT, Evans Fl. Pharmacological Methods in Phytotherapy Research. Vol. 1, Selection Prep Pharmacol Evaluat Plant Mat. England: Wiley and Sons; 1996. p. 228.

70. Adeneye AA, Olagunju JA. Preliminary hypoglycemic and hypolipidemic activities of the aqueous seed extract of Carica papaya Linn in Wistar rats. Biol Med. 2009. https://doi.org/10.4172/0974-8369.1000001.
71. Amit SK, Uddin MM, Rahman R, Islam SR, Khan MS. A review on mechanisms and commercial aspects of food preservation and processing. Agric Food Sec. 2017;6(1). https://doi.org/10.1186/s40066-017-0130-8.

72. Qiu L, Zhang M, Tang J, Adhikari B, Cao P. Innovative technologies for producing and preserving intermediate moisture foods: a review. Food Res Int. 2019;116:90-102. https://doi.org/10.1016/j.foodres.2018.12.055.

73. Akubor PI. Functional properties and performance of cowpea/plantain/ wheat flour blends in biscuits. Plant Foods Hum Nutr. 2003;58(3):1-8. https://doi.org/10.1023/B:QUAL.0000041154.09382.d8.

74. Adegunwa MO, Adelekan EO, Adebowale AA, Bakare HA, Alamu EO. Evaluation of nutritional and functional properties of plantain (Musa paradisiaca L.) and tigernut (Cyperus esculentus L.) flour blends for food formulations. Cog Chem. 2017;3(1). https://doi.org/10.1080/23312009.201 7.1383707 .

75. Anderson JW, Baird P, Davis RH, Ferreri S, Knudtson M, Koraym A, et al. Health benefits of dietary fiber. Nutr Rev. 2009;67(4):188-205. https://doi. org/10.1111/j.1753-4887.2009.00189.x.

76. Timm DA, Slavin JL. Dietary fiber and the relationship to chronic diseases. Am J Lifestyle Med. 2008;2(3):233-40. https://doi.org/10.1177/155982 7608314149.

77. Morakinyo AO, Samuel TA, Adegoke OA. Mineral composition of commonly consumed local foods in Nigeria. Afr J Biomed Res. 2016;19(Suppl 2):141-7.

78. Floegel A, Stefan N, Yu Z, Mühlenbruch K, Drogan D, Joost HG, et al. Identification of serum metabolites associated with risk of type 2 diabetes using a targeted metabolomic approach. Diabetes. 2013;62(2):639-48. https://doi.org/10.2337/db12-0495.

79. Tejero J, Biswas A, Wang ZQ, Page RC, Haque MM, Hemann C, et al. Stabilization and characterization of a heme-oxy reaction intermediate in inducible nitric-oxide synthase. J Biol Chem. 2008;283(48):33498-507. https://doi.org/10.1074/jbc.M806122200.

80. Harvey AL. Natural products in drug discovery. Drug Discov Today. 2008; 13(19-20):894-901. https://doi.org/10.1016/j.drudis.2008.07.004.

81. Shahidi F, Chandrasekara A. The use of antioxidants in the preservation of cereals and low-moisture foods. In Handbook of Antioxidants for Food Preservation (pp. 413-432): Woodhead Publishing; 2015. https://doi.org/10.1 016/B978-1-78242-089-7.00017-8.

82. Jeong JM, Choi $\mathrm{CH}$, Kang SK, Lee $\mathrm{H}$, Lee JY, Jung H. Antioxidant and chemosensitizing effects of flavonoids with hydroxy and/or methoxy groups and structure-activity relationship. J Pharm Pharm Sci. 2007. https:// doi.org/10.18433/J3KW2Z.

83. Chen GL, Zhang X, Chen SG, Han MD, Gao YQ. Antioxidant activities and contents of free, esterified and insoluble-bound phenolics in 14 subtropical fruit leaves collected from the south of China. J Funct Foods. 2017:30:290302. https://doi.org/10.1016/j.jf.2017.01.011.

84. Defrancesco $E$, Trestini $S$. The role of the name of the vine in the enhancement of a wine on the consumer market: first results of a survey on Tocai. 2008; https://agriregionieuropa.univpm.it/it/content/article/31/12/ilruolo-del-nome-del-vitigno-nella-valorizzazione-di-un-vino-sul-mercato-al

85. Brewer MS. Natural antioxidants: sources, compounds, mechanisms of action, and potential applications. Comprehens Rev Food Sci Food Saf. 2011;10(4):221-47. https://doi.org/10.1111/j.1541-4337.2011.00156.x.

86. Sanches-Silva A, Costa D, Albuquerque TG, Buonocore GG, Ramos F, Castilho MC, et al. Trends in the use of natural antioxidants in active food packaging: a review. Food Addit Contam A. 2014;31(3):374-95. https://doi. org/10.1080/19440049.2013.879215.

87. Lobo V, Patil A, Phatak A, Chandra N. Free radicals, antioxidants and functional foods: impact on human health. Pharmacog Rev. 2010;4(8):11826. https://doi.org/10.4103/0973-7847.70902.

88. Butt MS, Rizwana B. Nutritional and functional properties of some promising legumes protein isolates. Pak J Nutr. 2010;9(Suppl 4):373-9. https://doi.org/1 0.3923/pjn.2010.373.379

89. Theodore IM, Ikenebomeh MJ, Ezeibe S. Evaluation of mineral content and functional properties of fermented maize (generic and specific) flour blended with Bambara groundnut (Vigna subterranean). Afr J Food Sci. 2009;3:107-12.

90. Jude-Ojei BS, Lola A, Ajayi IO, Seun I. Functional and pasting properties of maize 'Ogi' supplemented with fermented Moringa seeds. J Food Proc Technol. 2017;08(05). https://doi.org/10.4172/2157-7110.1000674.

91. Lorenz K, Collins F. Quinoa, starch physicochemical properties and functional characteristics of starch. Starke. 1980:42(3):81-6. https://doi.org/1 0.1002/star.19900420302 
92. Akintayo ET, Oshodi AA, Esuoso KO. Effects of $\mathrm{NaCl}$, ionic strength and $\mathrm{pH}$ on the foaming and gelation of pigeon pea (Cajanus cajan) protein concentrates. Food Chem. 1999;66(1):51-6. https://doi.org/10.1016/503088146(98)00155-1.

93. Kaushal P, Kumar V, Sharma HK. Comparative study of physicochemical, functional, antinutritional and pasting properties of taro (Colocasia esculenta), rice (Oryza sativa) flour, pigeonpea (Cajanus cajan) flour and their blends. LWT-Food Sci Technol. 2012;48(1):59-68. https://doi.org/10.1016/j. Iwt.2012.02.028.

94. Chandra S, Singh S, Kumari D. Evaluation of functional properties of composite flours and sensorial attributes of composite flour biscuits. J Food Sci Technol. 2015. https://doi.org/10.1007/s13197-014-1427-2.

95. Onweluzo JC, Nnamuchi OM. Production and evaluation of porridge-type breakfast product from Treculia africana and Sorghum bicolor flours. Pak J Nutr. 2009.

96. Ajanaku KO, Ajanaku CO, Edobor-Osoh A, Nwinyi OC. Nutritive value of sorghum ogi fortified with groundnut seed (Arachis) hypogaea. Nutritive Value of Sorghum Ogi Fortified with Groundnut Seed (Arachis) hypogaea. Am J Food Technol. 2012;7(Suppl 2):82-8.

97. Danbaba N, Anounye JC, Gana AS, Abo ME, Ukwungwu MN, Maji AT. Physical and pasting properties of 'ofada' rice (Oryza sativa L.) varieties. Nig Food J. 2012;30(1):18-25. https://doi.org/10.1016/50189-7241(15)30009-6.

98. Kaur M, Shandu KS, Singh N. Comparative study of the functional, thermal and pasting properties of flour from different chickpea cultivars. J Food Chem. 2007;104(1):259-67. https://doi.org/10.1016/j.foodchem.2006.11.037.

99. Adebowale AA, Sanni LO, Awonorin SO. Effect of texture modifiers on the physicochemical and sensory properties of dried fufu. Food Sci Technol Int. 2005;11(5):373-82. https://doi.org/10.1177/1082013205058531.

100. Arinola SO, Ogunbusola EM, Adebayo SF. Effect of drying methods on the chemical, pasting and functional properties of unripe plantain (Musa paradisiaca) flour. Curr J Appl Sci Technol. 2016;14(3):1-7. https://doi.org/10. 9734/BJAST/2016/22936.

101. Shittu TA, Lasekan OO, Sanni LO, Oladosu MO. The effect of drying methods on the functional and sensory characteristics of pukuru-a fermented cassava product. ASSET Int J. 2001;1(Suppl 2):9-16.

102. Sandhu KS, Singh N, Malhi NS. Some properties of corn grains and their flours I: physicochemical, functional and chapati-making properties of flours. Food Chem. 2007;101(3):938-46. https://doi.org/10.1016/j. foodchem.2006.02.040

103. Sandhu KS, Singh N, Malhi NS. Physicochemical and thermal properties of starches separated from corn produced from crosses of two germ pools. Food Chem. 2005;89(4):541-8. https://doi.org/10.1016/j. foodchem.2004.03.007.

104. Okoh SO, Oyewole AO, Ishola RO, Odusote AD, Okoh OO, Igwe CC, et al. Antioxidant and free radical scavenging capacity of crude and refined oil extracted from Azadirachta indica a. Juss Int J Biol. 2015;7(2). https://doi. org/10.5539/ijb.v7n2px.

105. Kalpana S, Neelam C, Parul S, Kamini S. Effect of domestic methods of processing on nutrient composition of oat (Avena sativa) flour. Food Sci Res J. 2011;2(Suppl 2):116-21.

106. Friedman M. Rice brans, rice bran oils, and rice hulls: composition, food and industrial uses, and bioactivities in humans, animals, and cells. J Agri Food Chem. 2013;61(45):10626-41. https://doi.org/10.1021/j4403635v.

107. Zamindar N, Bashash M, Khorshidi F, Serjouie A, Shirvani MA, Abbasi H, et al. Antioxidant efficacy of soybean cake extracts in soy oil protection. J Food Sci Technol. 2017;54(7):2077-84. https://doi.org/10.1007/s13197-017-2646-0.

108. Chu YF, Sun JIE, Wu X, Liu RH. Antioxidant and antiproliferative activities of common vegetables. J Agric Food Chem. 2002;50(23):6910-6. https://doi. org/10.1021/jf020665f.

109. Ademiluyi AO, Akpambang VOE, Oboh G. Polyphenol contents and antioxidant capacity of tropical clove bud (Eugenia aromatica Kuntze). Lar Ital Dell Sost Gras. 2009;86(Suppl 2):131-7.

110. Lee RK, Cicchetti D, Carlson EA, Egeland B, Andrew CW. Genetic contributions to continuity and change in attachment security: a prospective, longitudinal investigation from infancy to young adulthood. Child Psychol Psychiat. 2013;54(Suppl 11):1223-30. https://doi.org/10.1111/ jcpp.12093.

111. Hasanein P, Shahidi S. Preventive effect of Teucrium polium on learning and memory deficits in diabetic rats. Med Sci Monit Int Med J Experim Clin Res. 2012. https://doi.org/10.12659/MSM.882201.
112. Gylling H, Tuominen JA, Koivisto VA, Miettinen TA. Cholesterol metabolism in type 1 diabetes. Diabetes. 2004;53(9):2217-22. https://doi.org/10.2337/dia betes.53.9.2217.

113. Martirosyan DM, Miroshnichenko LA, Kulakova SN, Pogojeva AV, Zoloedov VI. Amaranth oil application for coronary heart disease and hypertension. Lipids Health Dis. 2007;6(1):1. https://doi.org/10.1186/1476-511X-6-1.

114. Shen GX. Lipid disorders in diabetes mellitus and current management. Curr Pharm Analy. 2007;3(1):17-24. https://doi.org/10.2174/157341207779802386.

115. von Eckardstein A, Hersberger M, Rohrer L. Current understanding of the metabolism and biological actions of HDL. Curr Opin Clin Nutr Metab Care. 2005;8(Suppl 2):147-52. https://doi.org/10.1097/00075197-200503000-00007.

116. Kavey REW, Daniels SR, Lauer RM, Atkins DL, Hayman LL, Taubert K. American Heart Association guidelines for primary prevention of atherosclerotic cardiovascular disease beginning in childhood. Circulat. 2003:107(Suppl 11):1562-6

117. Pearson TA, Blair SN, Daniels SR, Eckel RH, Fair JM, Fortmann SP, et al. AHA guidelines for primary prevention of cardiovascular disease and stroke: 2002 update: consensus panel guide to comprehensive risk reduction for adult patients without coronary or other atherosclerotic vascular diseases. Circulat. 2002;106(3):388-91. https://doi.org/10.1161/01.CIR.0000020190.

118. NIS Nigerian Industrial Standard (NIS 344: 2004). Standard for Edible Cassava Flour. Lagos Nigeria: Standard Organisation of Nigeria; 2004. p. 12-34.

119. FAO/WHO. CODEX CAC/GL 08, 1991. Codex Alimentarius: Guidelines on Formulated Supplementary Foods for Older Infants and Young Children. (4): FAO/WHO Joint Publications; 1991. p. 144.

120. FAO/WHO/UNU. Protein and amino acid requirements in human nutrition: WHO Press; 2007. p. 150.

121. Morris ER, Ellis R. Bioavailability of dietary calcium: effect of phytate on adult men consuming non-vegetarian diets. ACS Sympos Ser. 1985. https://doi. org/10.1021/bk-1985-0275.ch006.

122. Hurrell RF. Influence of vegetable protein sources on trace element and mineral bioavailability. J Nutr. 2003;133(9):2973S-7S. https://doi.org/10.1093/ in/133.9.2973S.

123. Hemalatha S, Platel K, Srinivasan K. Zinc and iron contents and their bioaccessibility in cereals and pulses consumed in India. Food Chem. 2007; 102(4):1328-36. https://doi.org/10.1016/j.foodchem.2006.07.015.

124. American Diabetes Association. Glycemic targets: standards of medical care in diabetes-2019. Diabetes care. 2019. https://doi.org/10.2337/dc19-S006.

\section{Publisher's Note}

Springer Nature remains neutral with regard to jurisdictional claims in published maps and institutional affiliations.

\section{Submit your manuscript to a SpringerOpen ${ }^{\circ}$ journal and benefit from:}

- Convenient online submission

- Rigorous peer review

- Open access: articles freely available online

- High visibility within the field

- Retaining the copyright to your article

Submit your next manuscript at $\boldsymbol{\nabla}$ springeropen.com 